\title{
Convex analysis on polyhedral spaces
}

\author{
Ana María Botero ${ }^{1}$ - José Ignacio Burgos Gil ${ }^{2}$ Martín Sombra ${ }^{3,4,5}$
}

Received: 16 January 2020 / Accepted: 9 September 2021 / Published online: 24 January 2022

(c) The Author(s) 2022

\begin{abstract}
We introduce notions of concavity for functions on balanced polyhedral spaces, and we show that concave functions on such spaces satisfy several strong continuity properties.
\end{abstract}

Keywords Convex analysis · Polyhedral spaces · Tropical geometry

Mathematics Subject Classification Primary 26B25; Secondary 52B70 $\cdot 14$ T05

\section{Contents}

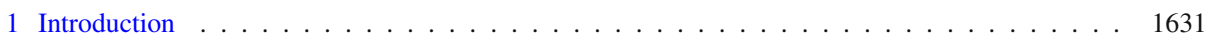

2 Polyhedral spaces . . . . . . . . . . . . . . . . . . . . . . . . . . . . . . 1634

3 Minkowski weights and Minkowski cycles . . . . . . . . . . . . . . . . . . . . . . . 1639

4 Concave piecewise affine functions on polyhedral spaces . . . . . . . . . . . . . . . . . . . 1647

5 Concave functions on polyhedral spaces . . . . . . . . . . . . . . . . . . . . . 1658

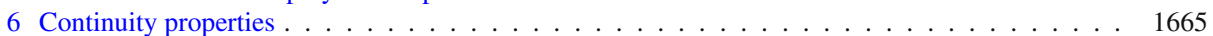

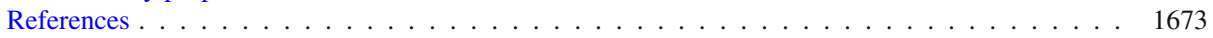

\section{Introduction}

Convex analysis studies properties of convex functions and convex sets. The notion of convexity is a simple and natural one which has been increasingly important in both pure and applied mathematics. One of the main advantages of working with convex functions is that they have nice topological properties. For instance, a convex function on a open subset $U \subset \mathbb{R}^{n}$ is

Botero was partially supported by the SFB Higher Invariants at the University of Regensburg. Burgos was partially supported by the MINECO research projects MTM2016-79400-P and PID2019-108936GB-C21 and by the Severo Ochoa programs for centers of excellence SEV-2015-0554 and CEX2019-000904-S (ICMAT Severo Ochoa). Sombra was partially supported by the MINECO research projects MTM2015-65361-P and PID2019-104047GB-I00 and by the María de Maeztu program for centers and units of excellence MDM-2014-0445 and CEX2020-001084-M.

Martín Sombra

sombra@ub.edu

http://www.maia.ub.edu/ sombra

Extended author information available on the last page of the article 
continuous on $U$, and Lipschitz continuous on every compact subset of it. The aim of this article is to transfer the notion of convexity of functions to balanced polyhedral spaces, and to extend to this setting the strong continuity properties of convex functions on open subsets of $\mathbb{R}^{n}$.

There are algebraic objects to which one can naturally attach a balanced polyhedral space. The results of this article can be applied directly to such spaces, and we hope that this serves to a better understanding of the algebraic objects involved. Important examples arise in tropical geometry. Here, the procedure of tropicalization attaches a tropical cycle to an algebraic cycle, and the tropical cycle has a structure of a balanced polyhedral space [1].

Related examples arise in the theory of toroidal embeddings. As a particular case, to a pair $(X, D)$ consisting of an algebraic variety $X$ and a simple normal crossings divisor $D$ on $X$ one can associate its Clemens complex (also called the dual complex), which is also a balanced polyhedral space [2,9].

In [1], Allerman and Rau developed an intersection theory between piecewise affine functions and tropical cycles. This theory was recently extended by Gross to a tropical intersection theory on weakly embedded conical polyhedral complexes associated to toroidal embeddings, between combinatorially principal piecewise affine functions and tropical cycles [9].

A balanced polyhedral space $X$ is an (abstract) polyhedral space of pure dimension $n$, which we equip with additional structure that allows to define a balancing condition. More precisely, it can be identified with a 5 -tuple $(X, \Pi, N, \iota, b)$, where $X$ is a second countable $n$-dimensional topological space, $\Pi$ is a polyhedral complex on $X, N$ is a Euclidean vector space, $\iota$ is a continuous map $\iota: X \rightarrow N$ whose restriction to each polyhedron $\sigma \in \Pi$ is injective and affine, and $b$ is an $n$-dimensional Minkowski weight on $\Pi$ satisfying $b(\sigma)>0$ for every $n$-dimensional cone $\sigma \in \Pi$. The map $\iota$ is called the quasi-embedding and $b$ the balancing condition (Definition 3.24).

The additional structure considered in the present article differs from the ones considered in [1] and [9] in the following two aspects: on the one side, the authors in loc. cit. consider an integral structure whereas in this article, a Euclidean structure is considered. This turns out to be more convenient when dealing with convexity notions. On the other side, a tropical variety as in [1] comes with an embedding into a real vector space, while a weak embedding as in [9] does not need to be injective when restricted to a cone. By contrast, a quasi-embedding does not need to be globally injective but its restriction to every polyhedron is assumed to be injective. A consequence of this difference is that we do not have to care about piecewise affine functions being combinatorially principal when doing intersection theory. Indeed, injectivity on each polyhedra implies that any piecewise affine function on the complex is combinatorially principal.

We will work with concave functions instead of convex ones, because the applications we have in mind come from toroidal geometry and positive divisors in this theory are described by concave functions on balanced polyhedral spaces [2]. Indeed, the functions associated to positive divisors in toric geometry are concave in the sense of convex analysis, although they are usually called convex in the toric literature [7].

A first approach to concavity on polyhedral spaces is to declare that a function $f$ on a balanced polyhedral space $X$ is concave if it is the pullback by the quasi-embedding $\iota$ of a concave function on the Euclidean space $N$. But this notion of concavity is not optimal because it is not local (Example 4.3). The variant that declares $f$ to be concave if it is locally of the above type, is not stable under the operation of taking the infimum of a family of such functions. More crucially, given a toroidal embedding, the piecewise affine function on the Clemens complex associated to a nef toroidal divisor on the algebraic variety is not necessarily concave in this sense. 
Hence it makes sense to explore other notions of concavity. In this article, we give several notions of concavity in terms of convex combinations. A convex combination of points in $X$ is a triple

$$
\left(x,\left\{x_{i}\right\}_{i \in I},\left\{v_{i}\right\}_{i \in I}\right)
$$

where $I$ is a finite set, $x \in X$ is the central point, the $x_{i}$ 's are also points in $X$ and the $v_{i}$ 's are nonnegative real numbers such that

$$
\sum_{i \in I} v_{i}=1 \quad \text { and } \quad \iota(x)=\sum_{i \in I} v_{i} \iota\left(x_{i}\right) .
$$

A convex combination is polyhedral if there is a polyhedral structure $\Pi$ on $X$ such that the central point $x$ belongs to a polyhedron $\tau$ and the remaining points belong to polyhedra having $\tau$ as a face. Finally, a convex combination is balanced if it is polyhedral and, roughly speaking, the location of the $x_{i}$ 's is dictated by the balancing condition (Definition 5.1).

Then, a function $f$ on $X$ is strongly concave if for all convex combinations, the concavity condition

$$
f(x) \geq \sum_{i \in I} v_{i} f\left(x_{i}\right)
$$

is satisfied. This is equivalent to asking that $f$ is the pullback by the quasi-embedding $\iota$ of a concave function on the Euclidean space $N$ (Proposition 5.7). The function $f$ is concave if the concavity condition (1.1) is satisfied for all polyhedral convex combination, and it is weakly concave if this concavity condition is only assumed to hold for balanced convex combinations (Definition 5.5).

Clearly, strongly concave functions are concave and, in turn, concave functions are weakly concave. The three notions of concavity are different (Examples 4.17, 4.18 and 4.19) but, when restricted to $\mathbb{R}^{n}$, they all agree with the usual one. Moreover, in the piecewise affine case, these notions of concavity can be reformulated in terms of intersection products with Minkowski cycles (Proposition 5.7).

The main results of this article show that weakly concave functions (hence also concave and strongly concave functions) on a balanced polyhedral space satisfy the same nice continuity properties satisfied by concave functions on $\mathbb{R}^{n}$.

The first result in this direction is Theorem 6.2 that states that, if $f$ is a weakly concave function on an open subset $U \subset X$, then it is continuous. Even more, in Corollary 6.22 we see that such a function is Lipschitz continuous in every compact subset $K \subset U$. More precisely, the Lipschitz constant of $f$ in $K$ can be bounded in terms of the sup-norm of the function on a slightly bigger open subset (Theorem 6.18).

Once we have proven Lipschitz continuity on compact subsets, we can prove some strong uniformity and convergence results for families of weakly concave functions (Theorems $6.23,6.24$ and 6.25). For instance, Theorem 6.24 states that, if $\left(f_{i}\right)_{i \geq 0}$ is a sequence of weakly concave functions that converge pointwise on a dense subset to finite limits, then the sequence of functions converges pointwise to a weakly concave function and the convergence is uniform on compact subsets.

As we mentioned earlier, our motivation comes from toroidal geometry. In the subsequent article by the first and the second authors [2], the strong continuity properties of concave functions in open subsets of polyhedral complexes are used to show that the degree of a nef toroidal b-divisor is well-defined and to prove a Hilbert-Samuel type theorem for such b-divisors. However, we believe that the results of this article are general enough to be of interest in other areas of mathematics. 
The article is organized as follows. In Sect. 2 we gather several definitions concerning polyhedral spaces, including the key notions of quasi-embedded and of Euclidean polyhedral spaces and complexes. We define morphisms between these spaces and show that they satisfy nice topological properties (Proposition 2.9).

In Sect. 3 we introduce the space of Minkowski weights on a Euclidean polyhedral complex (Definition 3.3) and the space of Minkowski cycles on a Euclidean polyhedral space (Definition 3.23). We also introduce balanced polyhedral spaces, that is, Euclidean polyhedral spaces endowed with a full-dimensional strictly positive Minkowski cycle (Definition 3.24). We end this section by defining an intersection product between Minkowski cycles and piecewise affine functions, and we show that it is well-defined and symmetric (Proposition 3.15). This intersection product is the Euclidean translation of that in [11] and [9].

In Sect. 4 we discuss different notions of concavity for piecewise affine functions related to preserving the positivity of the intersection product. We end this section by introducing the notions of regular polyhedral structures on a balanced polyhedral space $X$ and of strictly concave function. This is inspired by the correspondence between normal fans of polytopes and projective toric varieties. We then show the analogue of the toric Chow lemma from toric geometry, providing existence of regular structures whenever $X$ satisfies a natural finiteness condition (Theorem 4.34).

In Sect. 5 we discuss the different notions of concavity of functions on $X$ that are not necessarily piecewise affine, in terms of convex combinations of points. We show that in the piecewise affine case, these notions agree with those introduced in the previous section (Proposition 5.7).

Finally, in Sect. 6 we prove the strong continuity properties of weakly concave (and hence also of concave and strongly concave) functions on a balanced polyhedral space.

\section{Polyhedral spaces}

In this section we gather several definitions concerning polyhedral and complexes. For convex sets and functions we will use the notations and definitions in [12]. In particular, the empty set is a face of every convex set.

Definition 2.1 Let $X$ be a second countable topological space. A polyhedral structure on $X$ is a pair

$$
\Pi=\left(\left\{\sigma_{\alpha}\right\}_{\alpha \in \Lambda},\left\{M_{\alpha}\right\}_{\alpha \in \Lambda}\right)
$$

consisting of

(1) a collection $\left\{\sigma_{\alpha}\right\}_{\alpha \in \Lambda}$ of different closed subsets giving a locally finite covering of $X$,

(2) a collection $\left\{M_{\alpha}\right\}_{\alpha \in \Lambda}$ where each $M_{\alpha}$ is a finite dimensional vector space of continuous $\mathbb{R}$-valued functions on $\sigma_{\alpha}$ such that, setting $N_{\alpha}=\operatorname{Hom}\left(M_{\alpha}, \mathbb{R}\right)$, the evaluation map

$$
\phi_{\alpha}: \sigma_{\alpha} \longrightarrow N_{\alpha}
$$

sends $\sigma_{\alpha}$ homeomorphically onto a full-dimensional convex polyhedron of a hyperplane $H_{\alpha}$ of this dual vector space not passing through the origin.

The closed subsets $\sigma_{\alpha}$ are the polyhedra of $\Pi$, and the preimages under $\phi_{\alpha}$ of the faces of $\phi_{\alpha}\left(\sigma_{\alpha}\right)$ are the faces of $\sigma_{\alpha}$. We assume that the pair $\Pi$ satisfies the conditions:

(3) every face of a polyhedron $\sigma_{\alpha}$ of $\Pi$ is a polyhedron $\sigma_{\alpha^{\prime}}$ of $\Pi$ for an index $\alpha^{\prime} \in \Lambda$, and the corresponding vector space $M_{\alpha^{\prime}}$ consists of the restriction to $\sigma_{\alpha^{\prime}}$ of the functions in $M_{\alpha}$, 
(4) every two polyhedra of $\Pi$ intersect in a common face (possibly the empty one).

A polyhedral structure is finite if its set of polyhedra is finite.

For simplicity, we identify a polyhedral structure $\Pi$ with its underlying set of polyhedra. For a polyhedron $\sigma \in \Pi$ we write

$$
M_{\sigma}, \quad N_{\sigma}, \quad H_{\sigma} \quad \text { and } \phi_{\sigma}
$$

for its corresponding vector space, dual space, hyperplane and evaluation map, respectively.

Identifying each $\sigma \in \Pi$ with its image in $N_{\sigma}$, we can translate to it the objects and notions of polyhedra in vector spaces. In particular, we denote by relint $(\sigma)$ the relative interior of $\sigma$, that is, the preimage under $\phi_{\sigma}$ of the interior of the polyhedron $\phi_{\sigma}(\sigma) \subset N_{\sigma}$. Likewise the affine structure of $N_{\sigma}$ gives an affine structure on $\sigma$, and therefore we can talk about affine maps between polyhedra.

Remark 2.2 The condition (2) in Definition 2.1 implies that the space of affine functions on $\sigma$ coincides with $M_{\sigma}$.

Definition 2.3 Let $X$ be a second countable topological space and $\Pi, \Pi^{\prime}$ two polyhedral structures on $X$. Then $\Pi^{\prime}$ is a subdivision of $\Pi$, denoted by $\Pi^{\prime} \geq \Pi$, if for every $\sigma^{\prime} \in \Pi^{\prime}$ there exists a $\sigma \in \Pi$ with $\sigma^{\prime} \subset \sigma$, the inclusion being an affine map. Two polyhedral structures on $X$ are equivalent if they admit a common subdivision.

Proposition 2.4 Let X be a second countable topological space. Then

(1) the relation $\geq$ is a partial order on the set of polyhedral structures on $X$,

(2) the subdivisions of a given polyhedral structure on $X$ form a directed set,

(3) "being equivalent" is an equivalence relation between polyhedral structures on X.

Proof The statement in (1) is clear from the definitions. In particular, the antisymmetric property follows from the fact that the polyhedra in a given polyhedral structure are different, and so two polyhedral structures that subdivide each other are necessarily equal.

To prove the statement in (2), let $\Pi$ be polyhedral structure on $X$, and let $\Pi_{1}$ and $\Pi_{2}$ be two subdivisions of $\Pi$. For $i=1,2$ and each $\tau_{i} \in \Pi_{i}$ there is $\sigma_{i} \in \Pi$ such that $\tau_{i} \subset \sigma_{i}$. We have that $\sigma=\sigma_{1} \cap \sigma_{2}$ is a polyhedron of $\Pi$ containing the intersection $\tau_{1} \cap \tau_{2}$, and for each $i$ we have that $\tau_{i} \cap \sigma$ is a polyhedron of the vector space $N_{\sigma}$. Hence

$$
\tau_{1} \cap \tau_{2}=\left(\tau_{1} \cap \sigma\right) \cap\left(\tau_{2} \cap \sigma\right)
$$

is also a polyhedron of $N_{\sigma}$, and its space of affine functions $M_{\tau_{1} \cap \tau_{2}}$ consists of the functions in $M_{\sigma}$ restricted to it. The pair

$$
\left(\left\{\tau_{1} \cap \tau_{2}\right\}_{\tau_{1}, \tau_{2}},\left\{M_{\tau_{1} \cap \tau_{2}}\right\}_{\tau_{1}, \tau_{2}}\right)
$$

is a polyhedral structure on $X$ that subdivides both $\Pi_{1}$ and $\Pi_{2}$, and so the subdivisions of $\Pi$ form a directed set, as stated.

The relation "being equivalent" is clearly both symmetric and and reflexive. To check the transitivity property, let $\Pi, \Pi^{\prime}$ and $\Pi^{\prime \prime}$ be three polyhedral structures on $X$ such that $\Pi^{\prime}$ and $\Pi^{\prime \prime}$ are both equivalent to $\Pi$. Then there are polyhedral structures $\Pi_{1}$ and $\Pi_{2}$ that are a common subdivision of $\Pi$ and $\Pi^{\prime}$ and of $\Pi$ and $\Pi^{\prime \prime}$, respectively. Since $\Pi_{1}$ and $\Pi_{2}$ are subdivisions of $\Pi$, by (2) there is a further polyhedral structure $\Pi_{3}$ that subdivides both of them, and by (1) we have that $\Pi_{3}$ subdivides both $\Pi^{\prime}$ and $\Pi^{\prime \prime}$. Hence the later polyhedral structures are equivalent, proving (3). 
Definition 2.5 A polyhedral space $X$ is a second countable topological space equipped with an equivalence class of polyhedral structures. A polyhedral complex on $X$ is the choice of a representative of the class of polyhedral structures of $X$.

Remark 2.6 By Proposition 2.4, the set of polyhedral complexes on a polyhedral space is a directed set ordered by subdivision.

Definition 2.7 The dimension of a polyhedral space $X$ is defined as

$$
\operatorname{dim}(X)=\sup _{\sigma \in \Pi} \operatorname{dim}\left(M_{\sigma}\right)-1
$$

for any polyhedral complex $\Pi$ on $X$. We say that $X$ has pure dimension $n$ when every polyhedron of $\Pi$ that is maximal (with respect to the inclusion) has dimension $n$. These notions do not depend on the choice of $\Pi$.

Definition 2.8 Let $X$ and $X^{\prime}$ be polyhedral spaces. Given polyhedral complexes $\Pi$ on $X$ and $\Pi^{\prime}$ on $X^{\prime}$, a morphism of a polyhedral complexes between $\Pi$ and $\Pi^{\prime}$ is a continuous map $f: X \rightarrow X^{\prime}$ such that for every $\sigma \in \Pi$ there is $\sigma^{\prime} \in \Pi^{\prime}$ with $f(\sigma) \subset \sigma^{\prime}$, and the restriction $\left.f\right|_{\sigma}: \sigma \rightarrow \sigma^{\prime}$ is an affine map.

A morphism of polyhedral spaces between $X$ and $X^{\prime}$ is a continuous map $f: X \rightarrow X^{\prime}$ that becomes a morphism of polyhedral complexes after a suitable choice of polyhedral complexes $\Pi$ on $X$ and $\Pi^{\prime}$ on $X^{\prime}$ as above.

Proposition 2.9 The underlying topological space of a polyhedral space is Hausdorff.

Proof Let $X$ be a polyhedral space and $\Pi$ a polyhedral complex on $X$. Let $x, y \in X$ be two different points. Since the covering $\{\sigma\}_{\sigma \in \Pi}$ is locally finite, we can choose an open subset $W$ of $X$ containing both $x$ and $y$ and such that the set of polyhedra

$$
\Sigma=\{\sigma \in \Pi \mid \sigma \cap W \neq \emptyset\}
$$

is finite.

Each $\sigma \in \Sigma$ is homeomorphic to a polyhedron and so a Hausdorff topological subset of $X$. Hence there are open subsets $U_{\sigma}$ and $V_{\sigma}$ of $X$ that are disjoint on $\sigma$, and contain $x$ and $y$ respectively, and so

$$
W \cap \bigcap_{\sigma \in \Sigma} U_{\sigma} \quad \text { and } \quad W \cap \bigcap_{\sigma \in \Sigma} V_{\sigma}
$$

are disjoint neighborhoods of $x$ and $y$ respectively, proving the statement.

A standard way to construct polyhedral spaces is by gluing polyhedra through their faces, as we explain in the next example.

Example 2.10 Let $\Lambda$ be a countable set. For $\alpha \in \Lambda$, let $\Delta_{\alpha}$ be a polyhedron in a finite dimensional vector space $P_{\alpha}$. For each $\alpha, \beta \in \Lambda$, let $F_{\alpha, \beta}$ and $F_{\beta, \alpha}$ be faces of $\Delta_{\alpha}$ and $\Delta_{\beta}$ respectively (possibly the empty ones) and

$$
j_{\beta, \alpha}: F_{\alpha, \beta} \longrightarrow F_{\beta, \alpha}
$$

an affine isomorphism. We assume that this data verifies the conditions

(1) for $\alpha \in \Lambda, F_{\alpha, \alpha}=\Delta_{\alpha}$ and $j_{\alpha, \alpha}=\mathrm{id}_{\Delta_{\alpha}}$, 
(2) for $\alpha, \beta, \gamma \in \Lambda$, we have that $j_{\beta, \alpha}\left(F_{\alpha, \beta} \cap F_{\alpha, \gamma}\right)=F_{\beta, \alpha} \cap F_{\beta, \gamma}$ and

$$
j_{\gamma, \alpha}=j_{\gamma, \beta} \circ j_{\beta, \alpha} \text { on } F_{\alpha, \beta} \cap F_{\alpha, \gamma},
$$

(3) for $\alpha \in \Lambda$, the set $\left\{\beta \in \Lambda \mid F_{\beta, \alpha} \neq \emptyset\right\}$ is finite.

Note that (1) and (2) imply that, for $\alpha, \beta \in \Lambda$, we have that $j_{\alpha, \beta}=j_{\beta, \alpha}^{-1}$,

For $x \in \Delta_{\alpha}$ and $y \in \Delta_{\beta}$, we set $x \sim y$ whenever $x \in F_{\alpha, \beta}, y \in F_{\beta, \alpha}$ and $j_{\beta, \alpha}(x)=y$. This defines an equivalence relation on the disjoint union $\bigsqcup_{\alpha \in \Lambda} \Delta_{\alpha}$, and we consider the quotient topological space

$$
Y=\left(\bigsqcup_{\alpha \in \Lambda} \Delta_{\alpha}\right) / \sim .
$$

Since $Y$ is equipped with the quotient topology, the map $q: \bigsqcup_{\alpha \in \Lambda} \Delta_{\alpha} \rightarrow Y$ is continuous. The conditions above imply that the equivalence relation $\sim$ is closed and that the map $q$ is open. By [5, Chapter I, §8.3, Proposition 8] we deduce that $Y$ is Hausdorff. Moreover, the finiteness condition (3) implies that the quotient map $q$ is proper.

The image in $Y$ of each $\Delta_{\alpha}$ is a closed subset that is homeomorphic to it. Identifying each of these polyhedra with its image in $Y$ and defining $M_{\alpha}$ as the space of affine functions on $\Delta_{\alpha}$, we have that

$$
\Gamma=\left(\left\{\Delta_{\alpha}\right\}_{\alpha \in \Lambda},\left\{M_{\alpha}\right\}_{\alpha \in \Lambda}\right)
$$

is a polyhedral structure on $Y$ in the sense of Definition 2.1. Indeed, the condition (3) implies that the covering $\left\{\Delta_{\alpha}\right\}_{\alpha \in \Lambda}$ is locally finite.

Proposition 2.13 Every polyhedral complex on a polyhedral space is isomorphic to one constructed gluing polyhedra through faces as in Example 2.10.

Proof With notation as in Definition 2.1, let $\Pi=\left(\left\{\sigma_{\alpha}\right\}_{\alpha \in \Lambda},\left\{M_{\alpha}\right\}_{\alpha \in \Lambda}\right)$ be a polyhedral complex on a polyhedral space $X$. For each $\alpha \in \Lambda$ consider the polyhedron in the dual space $N_{\alpha}=\operatorname{Hom}\left(M_{\alpha}, \mathbb{R}\right)$ given by

$$
\Delta_{\alpha}=\phi_{\alpha}\left(\sigma_{\alpha}\right)
$$

and, for $\alpha, \beta \in \Lambda$, consider the faces of $\Delta_{\alpha}$ and $\Delta_{\beta}$ respectively defined as

$$
F_{\alpha, \beta}=\phi_{\alpha}\left(\sigma_{\alpha} \cap \sigma_{\beta}\right) \quad \text { and } \quad F_{\beta, \alpha}=\phi_{\beta}\left(\sigma_{\alpha} \cap \sigma_{\beta}\right)
$$

and the affine map $j_{\beta, \alpha}: F_{\alpha, \beta} \rightarrow F_{\beta, \alpha}$ given by the restriction of $\phi_{\beta} \circ\left(\phi_{\alpha}\right)^{-1}$ to the face $F_{\alpha, \beta}$.

This data satisfies the conditions in Example 2.10 and in particular, the hypothesis that the covering $\left\{\sigma_{\alpha}\right\}_{\alpha \in \Lambda}$ is locally finite implies the condition (3). Hence we can consider its associated polyhedral complex $\Gamma$ on the polyhedral space $Y$ as in (2.12) and (2.11). The isomorphisms $\phi_{\alpha}^{-1}: \Delta_{\alpha} \rightarrow \sigma_{\alpha}, \alpha \in \Lambda$, induce a bijective map

$$
f: Y \longrightarrow X
$$

that is continuous, by the universal property of the quotient topology.

Since the set of polyhedra $\sigma_{\alpha}, \alpha \in \Lambda$, forms a locally finite covering of $X$ by closed subsets and the restriction $\left.f^{-1}\right|_{\sigma_{\alpha}}=\phi_{\alpha}$ to each of them is continuous, the inverse map $f^{-1}$ is also continuous. Hence $f$ is a homeomorphism that is affine between each pair of polyhedra $\Delta_{\alpha}$ and $\sigma_{\alpha}$, and so it is an isomorphism of polyhedral complexes. 
To do convex analysis on a polyhedral space, we need a notion encoding how its different polyhedra are placed with respect to each other. For this, we first need to map it to a fixed ambient space. The definition below is a variant of [9, Definition 2.1].

Definition 2.14 A quasi-embedded polyhedral space is a triple $(X, N, \iota)$ where $X$ is a polyhedral space, $N$ a finite dimensional $\mathbb{R}$-vector space, and $\iota$ a map $X \rightarrow N$ such that $\iota(X)$ is contained in an affine hyperplane $H$ not containing zero and there is a polyhedral complex on $X$ for which the restriction of $\iota$ to each of its polyhedra is affine and injective. The map $\iota$ is called the quasi-embedding of $X$ in $N$.

A polyhedral complex $\Pi$ on the quasi-embedded polyhedral space $(X, N, \iota)$ is a polyhedral complex on $X$ satisfying the above condition, namely that $\iota$ is affine and injective on each of its polyhedra. For each $\sigma \in \Pi$, its image with respect to the evaluation map $\phi_{\sigma}$ spans a full-dimensional polyhedral cone of $N_{\sigma}$, and so the quasi-embedding $\iota$ induces a linear injective map

$$
\iota_{\sigma}: N_{\sigma} \rightarrow N
$$

that sends $H_{\sigma}$ to $H$.

We will usually denote a quasi-embedded polyhedral space by its underlying polyhedral space $X$ and, in this case, we will denote the corresponding quasi-embedding, vector space and hyperplane by $\iota_{X}, N_{X}$ and $H_{X}$, respectively.

Definition 2.15 A Euclidean polyhedral space is a quasi-embedded polyhedral space $X$ for which the vector space $N_{X}$ is equipped with a Euclidean metric.

A polyhedral complex on a Euclidean polyhedral space is a polyhedral complex $\Pi$ on the associated quasi-embedded polyhedral space. In this situation, for each polyhedron $\sigma \in \Pi$ the Euclidean metric on $N_{X}$ induces a Euclidean metric on the vector space $N_{\sigma}$.

Definition 2.16 A morphism between two quasi-embedded polyhedral spaces $f: X \rightarrow X^{\prime}$ is a pair $f=\left(f_{1}, f_{2}\right)$ where $f_{1}: X \rightarrow X^{\prime}$ is a morphism of polyhedral spaces and $f_{2}: N_{X} \rightarrow$ $N_{X^{\prime}}$ is an affine map such that the diagram

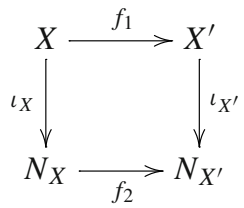

commutes. A morphism between Euclidean polyhedral spaces is a morphism between their underlying quasi-embedded polyhedral spaces.

Remark 2.17 The main differences between the notions of quasi-embedded polyhedral complex in Definition 2.14 and that of weakly embedded polyhedral complex in [9, Definition 2.1] are that for the latter, first the affine maps $\iota_{\sigma}$ are not required to be injective and second, the vector spaces $M$ and $M_{\sigma}$ are equipped with a lattice and the affine maps $\iota_{\sigma}$ are lattice maps. Here we shift the focus from lattices to Euclidean metrics because it is more convenient to do convex analysis. 


\section{Minkowski weights and Minkowski cycles}

In this section we introduce Minkowski weights on polyhedral complexes and Minkowski cycles on Euclidean polyhedral spaces. This will allow us to define balanced polyhedral spaces, the spaces on which we consider the different notions of concavity. We also define and study the basic operations on Minkowski weights and cycles, including their restriction to open subsets, pullback to subdivisions, and product with piecewise affine functions.

Throughout this section, we denote by $X$ a Euclidean polyhedral space and $U$ an open subset of it.

Definition 3.1 Let $\Pi$ be a polyhedral complex on $X$. The restriction of $\Pi$ to $U$, denoted by $\left.\Pi\right|_{U}$, is the set of polyhedra given by

$$
\left.\Pi\right|_{U}=\{\sigma \in \Pi \mid \sigma \cap U \neq \emptyset\} .
$$

For $k \in \mathbb{Z}_{\geq 0}$, we denote by $\left.\Pi\right|_{U}(k)$ the set of polyhedra of $\left.\Pi\right|_{U}$ of dimension $k$. The skeleton of $\left.\Pi\right|_{U}$ of dimension $k$, denoted by $\operatorname{Sk}_{k}\left(\left.\Pi\right|_{U}\right)$, is the subset of $X$ given by the union of these polyhedra.

The restriction of a polyhedral complex to an open subset is not a polyhedral complex, because it does not contain all the faces of its constituent polyhedra. For instance, if $X=[0,1]$ is the unit interval, $\Pi=\{[0,1],\{0\},\{1\}, \emptyset\}$ is the standard polyhedral complex on $[0,1]$ and $U=[0,1 / 2)$, then $\left.\Pi\right|_{U}=\{[0,1],\{0\}\}$ and the faces $\emptyset$ and $\{1\}$ of $[0,1]$ do not belong to $\left.\Pi\right|_{U}$.

Definition 3.2 Let $\Pi$ be a polyhedral complex on $X$ and let $\sigma, \tau \in \Pi$ such that $\tau$ is nonempty and a facet of $\sigma$, that is, a face of $\sigma$ of codimension 1. With notation as in Definition 2.14, the affine subspace $\iota_{X}\left(H_{\sigma}\right)$ of the Euclidean space $N_{X}$ contains $\iota_{X}\left(H_{\tau}\right)$ as a hyperplane. The unit vector normal to $\tau$ in the direction of $\sigma$, denoted by $v_{\sigma \backslash \tau}$, is defined as the unique unit vector in $N_{X}$ that is orthogonal to $\iota_{X}\left(H_{\tau}\right)$, parallel to $\iota_{X}\left(H_{\sigma}\right)$ and points towards $\sigma$ from $\tau$.

The next definition of Minkowski weights is the adaptation to our setting of the classical notion for lattice fans introduced by Fulton and Sturmfels in [8]. For $\sigma, \tau \in \Pi$, we write either $\tau \prec \sigma$ or $\sigma \succ \tau$ to indicate that $\tau$ is a face of $\sigma$.

Definition 3.3 Let $\Pi$ be a polyhedral complex on $X$ and $k \in \mathbb{Z}_{\geq 0}$. A weight on $\left.\Pi\right|_{U}$ of dimension $k$ is a map $c:\left.\Pi\right|_{U}(k) \rightarrow \mathbb{R}$. Its support is the subset of $X$ given by

$$
|c|=\bigcup_{c(\sigma) \neq 0} \sigma .
$$

This weight is positive if $c(\sigma) \geq 0$ for all $\left.\sigma \in \Pi\right|_{U}(k)$. For convenience, any weight $c$ on $\left.\Pi\right|_{U}$ of dimension $k$ is extended to a function $c:\left.\Pi\right|_{U} \rightarrow \mathbb{R}$ by setting $c(\sigma)=0$ for all $\left.\sigma \in \Pi\right|_{U}(\ell)$ with $\ell \neq k$.

A weight $c$ on $\left.\Pi\right|_{U}$ of dimension $k$ is a Minkowski weight if for each $\left.\tau \in \Pi\right|_{U}(k-1)$,

$$
\sum_{\sigma \in \prod_{\sigma \succ \tau}(k)} c(\sigma) v_{\sigma \backslash \tau}=0 .
$$

The set of weights on $\left.\Pi\right|_{U}$ of dimension $k$, denoted $W_{k}\left(\left.\Pi\right|_{U}\right)$, is an Abelian group under the addition of functions. The subgroup of its Minkowski weights is denoted by $M_{k}\left(\left.\Pi\right|_{U}\right)$, and the cone of those that are positive is denoted by $M_{k}^{+}\left(\left.\Pi\right|_{U}\right)$. For short, when $U=X$ we denote this Abelian group, subgroup and cone by $W_{k}(\Pi), M_{k}(\Pi)$ and $M_{k}^{+}(\Pi)$, respectively. 
Definition 3.5 A piecewise affine function on the open subset $U$ of the polyhedral space $X$ is a function $f: U \rightarrow \mathbb{R}$ for which there is a polyhedral complex $\Pi$ on $X$ such that for each $\left.\sigma \in \Pi\right|_{U}$ the restriction $\left.f\right|_{\sigma \cap U}$ is affine or equivalently, it is given by an element of $M_{\sigma}$. In this situation, we say that $f$ is defined on $\Pi$.

For each $\left.\sigma \in \Pi\right|_{U}$, we denote by $f_{\sigma}$ a linear function on $N_{X}$ satisfying

$$
\left.f\right|_{\sigma \cap U}=\left.f_{\sigma} \circ \iota_{X, \sigma}\right|_{\sigma \cap U},
$$

Since the image of $\sigma$ in $N_{\sigma}$ spans a full-dimensional polyhedral cone, the restriction of $f_{\sigma}$ to $\iota_{X, \sigma}\left(N_{\sigma}\right)$ does not depend on the choice of this linear function.

We denote by $\mathrm{PA}(U)$ the Abelian group of piecewise affine functions on $U$, and by $\operatorname{PA}_{\Pi}(U)$ the subgroup of those piecewise affine functions that are defined on $\Pi$.

Remark 3.6 Piecewise affine functions on polyhedral spaces are continuous, because they are continuous on the restriction to $U$ of each polyhedron of the polyhedral complex $\Pi$, and these polyhedra form a locally finite closed covering of the polyhedral space $X$.

We next define the basic operations on weights on polyhedral complexes and study their interplay.

Definition 3.7 (Restriction to open subsets) Let $\Pi$ be a polyhedral complex on $X, V$ an open subset of $U$, and $c$ a $k$-dimensional weight on $\left.\Pi\right|_{U}$. The restriction of $c$ to $V$, denoted by $\left.c\right|_{V}$, is the $k$-dimensional weight on $\left.\Pi\right|_{V}$ given by the restriction of this weight to the subset $\left.\Pi\right|_{V}$ of $\left.\Pi\right|_{U}$.

Definition 3.8 (Pullback to subdivisions) Let $\Pi, \Pi^{\prime}$ be polyhedral complexes on $X$ with $\Pi^{\prime} \geq \Pi$ and $c$ a $k$-dimensional weight on $\left.\Pi\right|_{U}$. The pullback of $c$ to $\Pi^{\prime}$, denoted by $c_{\Pi^{\prime}}$, is the $k$-dimensional weight on $\left.\Pi^{\prime}\right|_{U}$ defined, for $\left.\sigma^{\prime} \in \Pi^{\prime}\right|_{U}$, by

$$
c_{\Pi^{\prime}}\left(\sigma^{\prime}\right)= \begin{cases}c(\sigma) & \text { if there is }\left.\sigma \in \Pi\right|_{U} \text { with } \sigma \supset \sigma^{\prime} \text { and } \operatorname{dim}(\sigma)=\operatorname{dim}\left(\sigma^{\prime}\right), \\ 0 & \text { else. }\end{cases}
$$

Definition 3.9 (Product with piecewise affine functions) Let $\Pi$ be a polyhedral complex on $X, f$ a piecewise affine function on $U$ defined on $\Pi$, and $c$ a $k$-dimensional weight on $\left.\Pi\right|_{U}$. The product of $f$ and $c$, denoted by $f \cdot c$, is the $(k-1)$-dimensional weight on $\left.\Pi\right|_{U}$ defined, for each $\left.\tau \in \Pi\right|_{U}(k-1)$, by

$$
(f \cdot c)(\tau)=-\sum_{\sigma \succ \tau} c(\sigma) f_{\sigma}\left(v_{\sigma \backslash \tau}\right),
$$

the sum being over the $k$-dimensional polyhedra $\left.\sigma \in \Pi\right|_{U}$ having $\tau$ as a facet.

Choosing any point $x \in \tau$, the formula in (3.10) can be alternatively written as

$$
(f \cdot c)(\tau)=\left(\sum_{\sigma \succ \tau} c(\sigma)\right) f(x)-\sum_{\sigma \succ \tau} c(\sigma) f_{\sigma}\left(\iota_{X, \sigma}(x)+v_{\sigma \backslash \tau}\right) .
$$

Proposition 3.12 Let $\Pi$ be a polyhedral complex on $X, V$ an open subset of $U, \Pi^{\prime}$ a subdivision of $\Pi, f$ a piecewise affine function on $U$ defined on $\Pi$, and $c$ a weight on $\left.\Pi\right|_{U}$. Then

$$
\left.\left(c_{\Pi^{\prime}}\right)\right|_{V}=\left(\left.c\right|_{V}\right)_{\Pi^{\prime}},\left.\quad(f \cdot c)\right|_{V}=\left.\left.f\right|_{V} \cdot c\right|_{V} \quad \text { and } \quad(f \cdot c)_{\Pi^{\prime}}=f \cdot c_{\Pi^{\prime}}
$$


Proof The first equality, that is, the compatibility between the restriction to an open subset and the pullback to a subdivision, follows almost immediately from the definitions. Both $\left.\left(c_{\Pi^{\prime}}\right)\right|_{V}$ and $\left(\left.c\right|_{V}\right)_{\Pi^{\prime}}$ are $k$-dimensional weights on $\left.\Pi^{\prime}\right|_{V}$ and for each $\left.\sigma^{\prime} \in \Pi^{\prime}\right|_{V}$, their possibly nonzero values are respectively defined by

(1) $\left.\left(c_{\Pi^{\prime}}\right)\right|_{V}\left(\sigma^{\prime}\right)=c(\sigma)$ if there is $\left.\sigma \in \Pi\right|_{U}$ with $\sigma \supset \sigma^{\prime}$ and $\operatorname{dim}(\sigma)=\operatorname{dim}\left(\sigma^{\prime}\right)$,

(2) $\left(\left.c\right|_{V}\right)_{\Pi^{\prime}}\left(\sigma^{\prime}\right)=c(\widetilde{\sigma})$ if there is $\left.\widetilde{\sigma} \in \Pi\right|_{V}$ with $\widetilde{\sigma} \supset \sigma^{\prime}$ and $\operatorname{dim}(\widetilde{\sigma})=\operatorname{dim}\left(\sigma^{\prime}\right)$.

Since $\sigma^{\prime} \cap V \neq \varnothing$ and $\sigma^{\prime} \supset \sigma$, we have that $\left.\sigma \in \Pi\right|_{V}$. Hence $\sigma=\widetilde{\sigma}$ and both weights coincide, as stated.

The second equality is also direct from the definitions, since the product of a weight with a piecewise affine function is defined in local terms.

Hence we turn to the third equality, giving the compatibility between the pullback to a subdivision and the product with a piecewise affine function. Both $(f \cdot c)_{\Pi^{\prime}}$ and $f \cdot c_{\Pi^{\prime}}$ are $(k-1)$-dimensional weights on $\left.\Pi^{\prime}\right|_{U}$, and so it is enough to consider their values on the set of polyhedra $\left.\Pi^{\prime}\right|_{U}(k-1)$.

Let $\left.\tau^{\prime} \in \Pi^{\prime}\right|_{U}(k-1)$ and denote by $\tau$ the minimal polyhedron in $\left.\Pi\right|_{U}$ containing $\tau^{\prime}$. On the one hand, if $\operatorname{dim}(\tau)=k-1$ then, with notation as in Definition 3.9,

$$
(f \cdot c)_{\Pi^{\prime}}\left(\tau^{\prime}\right)=(f \cdot c)(\tau)=-\sum_{\sigma \in \prod_{\sigma \succ \tau}(k)} c(\sigma) f_{\sigma}\left(v_{\sigma \backslash \tau}\right),
$$

whereas if $\operatorname{dim}(\tau) \geq k$ then $(f \cdot c)_{\Pi^{\prime}}\left(\tau^{\prime}\right)=0$. On the other hand,

$$
\left(f \cdot c_{\Pi^{\prime}}\right)\left(\tau^{\prime}\right)=-\sum_{\substack{\left.\sigma^{\prime} \in \Pi^{\prime}\right|_{U}(k) \\ \sigma^{\prime} \succ \tau^{\prime}}} c_{\Pi^{\prime}}\left(\sigma^{\prime}\right) f_{\sigma^{\prime}}\left(v_{\sigma^{\prime} \backslash \tau^{\prime}}\right) .
$$

When $\operatorname{dim}(\tau) \geq k+1$, for each $\left.\sigma^{\prime} \in \Pi^{\prime}\right|_{U}(k)$ with $\sigma^{\prime} \succ \tau^{\prime}$ the minimal polyhedron $\left.\sigma \in \Pi^{\prime}\right|_{U}$ containing it also contains $\tau$ and so it has dimension at least $k+1$. Hence $c_{\Pi^{\prime}}\left(\sigma^{\prime}\right)=0$ and the formula in (3.14) implies that $\left(f \cdot c_{\Pi^{\prime}}\right)\left(\tau^{\prime}\right)=0$, proving the equality in this case.

When $\operatorname{dim}(\tau)=k$, there are two polyhedra $\sigma^{\prime},\left.\sigma^{\prime \prime} \in \Pi^{\prime}\right|_{U}(k)$ contained in $\tau$ and having $\tau^{\prime}$ as a facet. We have that

$$
c_{\Pi^{\prime}}\left(\sigma^{\prime}\right)=c_{\Pi^{\prime}}\left(\sigma^{\prime \prime}\right) \quad \text { and } \quad v_{\sigma^{\prime} \backslash \tau^{\prime}}=-v_{\sigma^{\prime \prime} \backslash \tau^{\prime}},
$$

and also that $f_{\sigma^{\prime}}$ and $f_{\sigma^{\prime \prime}}$ coincide with $f_{\tau}$ on $\iota_{X, \sigma}\left(N_{\sigma}\right)=\iota_{X, \sigma^{\prime}}\left(N_{\sigma^{\prime}}\right)=\iota_{X, \tau}\left(N_{\tau}\right)$. Hence, the contributions of these two polyhedra to the sum in (3.14) cancel. For any other polyhedron in $\left.\Pi^{\prime}\right|_{U}(k)$ having $\tau^{\prime}$ as a facet, the minimal polyhedron in $\left.\Pi\right|_{U}$ containing it has dimension greater than $k$ and so its value for the weight $c_{\Pi^{\prime}}$ is zero. Thus again $\left(f \cdot c_{\Pi^{\prime}}\right)\left(\tau^{\prime}\right)=0$ in this case.

Finally suppose that $\operatorname{dim}(\tau)=k-1$. To each polyhedron $\left.\sigma^{\prime} \in \Pi^{\prime}\right|_{U}(k)$ contained in the $k$-dimensional skeleton $\operatorname{Sk}_{k}\left(\left.\Pi\right|_{U}\right)$ and having $\tau$ as a facet, we associate the minimal polyhedron $\left.\sigma \in \Pi\right|_{U}$ containing it. This assignment gives a bijection between this set of $k$-dimensional polyhedra of $\left.\Pi^{\prime}\right|_{U}$ and that of polyhedra in $\left.\Pi\right|_{U}(k)$ having $\tau$ as a facet, and we have that

$$
c_{\Pi^{\prime}}\left(\sigma^{\prime}\right)=c(\sigma) \quad \text { and } \quad v_{\sigma^{\prime} \backslash \tau^{\prime}}=v_{\sigma \backslash \tau}
$$

and also that $f_{\sigma^{\prime}}$ and $f_{\sigma^{\prime \prime}}$ coincide on $\iota_{X, \sigma}\left(N_{\sigma}\right)=\iota_{X, \sigma^{\prime}}\left(N_{\sigma^{\prime}}\right)$. Hence the sum in (3.14) coincides with that in (3.13), since the value of the weight $c_{\Pi^{\prime}}$ at the polyhedra in $\left.\Pi^{\prime}\right|_{U}(k)$ that are not contained in $\operatorname{Sk}_{k}\left(\left.\Pi\right|_{U}\right)$ is zero. Thus in this case $(f \cdot c)_{\Pi^{\prime}}\left(\tau^{\prime}\right)=f \cdot c_{\Pi^{\prime}}\left(\tau^{\prime}\right)$, which concludes the proof. 
We next prove that the product of several piecewise affine functions with a weight is commutative. This result is similar to [1, Proposition 3.7a], and its proof is done in a similar way.

Proposition 3.15 Let $\Pi$ be a polyhedral complex on $X, f, g$ piecewise affine functions on $U$ defined on $\Pi$, and c a $k$-dimensional weight on $\left.\Pi\right|_{U}$. Then

$$
f \cdot(g \cdot c)=g \cdot(f \cdot c) .
$$

Proof The proof is based on the following observation. Let $v_{1}$ and $v_{2}$ be two linearly independent unit vectors in a Euclidean space. Denote by $v_{1}^{\perp}$ and $v_{2}^{\perp}$ the unit vectors in the plane generated by $v_{1}$ and $v_{2}$, that are respectively orthogonal to $v_{1}$ and to $v_{2}$, and that both $v_{1}, v_{1}^{\perp}$ and $v_{2}^{\perp}, v_{2}$ have the same orientation as $v_{1}, v_{2}$. Then for $a, b \in \mathbb{R}$, the equations

$$
v_{1}^{\perp}=a v_{1}+b v_{2} \quad \text { and } \quad v_{2}^{\perp}=a v_{2}+b v_{1}
$$

are equivalent. Indeed, consider the reflection on the plane generated by $v_{1}$ and $v_{2}$ by the bisector of the angle between these two vectors. This reflection interchanges $v_{1}$ with $v_{2}$ and $v_{1}^{\perp}$ with $v_{2}^{\perp}$ and respects linear relations. Applying it to any of the two equations in (3.16) gives the other one, proving that they are equivalent.

Now let $\left.\rho \in \Pi\right|_{U}(k-2)$. For each $\left.\tau \in \Pi\right|_{U}(k-1)$ and $\left.\sigma \in \Pi\right|_{U}(k)$ with $\rho \prec \tau \prec \sigma$, we denote by $\tau^{\prime}$ the unique polyhedron different from $\tau$ that lies in $\left.\Pi\right|_{U}(k-1)$ and verifies that $\rho \prec \tau^{\prime} \prec \sigma$. The vectors $v_{\tau \backslash \rho}, v_{\tau^{\prime} \backslash \rho}, v_{\sigma \backslash \tau}$ and $v_{\sigma \backslash \tau^{\prime}}$ satisfy the conditions of the vectors $v_{1}$, $v_{2}, v_{1}^{\perp}$ and $v_{2}^{\perp}$ in the previous discussion, and so there are real numbers $a_{\sigma, \rho}$ and $b_{\sigma, \rho}$ such that

$$
v_{\sigma \backslash \tau}=a_{\sigma, \rho} v_{\tau \backslash \rho}+b_{\sigma, \rho} v_{\tau^{\prime} \backslash \rho} \quad \text { and } \quad v_{\sigma \backslash \tau^{\prime}}=a_{\sigma, \rho} v_{\tau^{\prime} \backslash \rho}+b_{\sigma, \rho} v_{\tau \backslash \rho}
$$

We compute

$$
\begin{aligned}
(f \cdot(g \cdot c))(\rho)= & \sum_{\tau \succ \rho}\left(\sum_{\sigma \succ \tau} c(\sigma) g_{\sigma}\left(v_{\sigma \backslash \tau}\right)\right) f_{\tau}\left(v_{\tau \backslash \rho}\right) \\
= & \sum_{\tau \succ \rho} \sum_{\sigma \succ \tau} c(\sigma) g_{\sigma}\left(a_{\sigma, \rho} v_{\tau \backslash \rho}+b_{\sigma, \rho} v_{\tau^{\prime} \backslash \rho}\right) f_{\tau}\left(v_{\tau \backslash \rho}\right) \\
= & \sum_{\sigma \succ \rho} c(\sigma) a_{\sigma, \rho}\left(\sum_{\sigma \succ \tau \succ \rho} f_{\tau}\left(v_{\tau \backslash \rho}\right) g_{\tau}\left(v_{\tau \backslash \rho}\right)\right) \\
& +\sum_{\sigma \succ \rho} c(\sigma) b_{\sigma, \rho}\left(\sum_{\sigma \succ \tau \succ \rho} f_{\tau}\left(v_{\tau \backslash \rho}\right) g_{\tau^{\prime}}\left(v_{\tau^{\prime} \backslash \rho}\right)\right),
\end{aligned}
$$

where the indexes $\tau$ and $\sigma$ go over the sets of polyhedra $\left.\Pi\right|_{U}(k-1)$ and $\left.\Pi\right|_{U}(k)$, respectively. The first equality is the definition of the product, the second follows from the first equation in (3.17), and the third comes from the linearity of $g_{\sigma}$ combined with the fact that $\left.g_{\sigma}\right|_{N_{\tau}}=g_{\tau}$ and $\left.g_{\sigma}\right|_{N_{\tau^{\prime}}}=g_{\tau^{\prime}}$.

Next, we interchange the roles of $\tau$ and $\tau^{\prime}$ in the inner sum of the second term of the last expression and revert the previous argument, applying this time the linearity of $f_{\sigma}$, the second equation in (3.17) and again the definition of the product to obtain that 


$$
\begin{aligned}
(f \cdot(g \cdot c))(\rho)= & \sum_{\sigma \succ \rho} c(\sigma) a_{\sigma, \rho}\left(\sum_{\sigma \succ \tau \succ \rho} f_{\tau}\left(v_{\tau \backslash \rho}\right) g_{\tau}\left(v_{\tau \backslash \rho}\right)\right) \\
& +\sum_{\sigma \succ \rho} c(\sigma) b_{\sigma, \rho}\left(\sum_{\sigma \succ \tau \succ \rho} f_{\tau^{\prime}}\left(v_{\tau^{\prime} \backslash \rho}\right) g_{\tau}\left(v_{\tau \backslash \rho}\right)\right) \\
= & \sum_{\tau \succ \rho} \sum_{\sigma \succ \tau} c(\sigma) f_{\sigma}\left(a_{\sigma, \rho} v_{\tau \backslash \rho}+b_{\sigma, \rho} v_{\tau^{\prime} \backslash \rho}\right) g_{\tau}\left(v_{\tau \backslash \rho}\right) \\
= & \sum_{\tau \succ \rho}\left(\sum_{\sigma \succ \tau} c(\sigma) f_{\sigma}\left(v_{\sigma \backslash \tau}\right)\right) g_{\tau}\left(v_{\tau \backslash \rho}\right) \\
= & (g \cdot(f \cdot c))(\rho),
\end{aligned}
$$

which proves the statement.

In view of the definition of the product, we give the following interpretation of the condition for an arbitrary weight to be a Minkowski weight.

Lemma 3.18 Let $\Pi$ be polyhedral complex on $X$ and c a weight on $\left.\Pi\right|_{U}$. Then c is a Minkowski weight if and only if for every linear function $\ell: N_{X} \rightarrow \mathbb{R}$,

$$
\left(\ell \circ \iota_{X}\right) \cdot c=0 .
$$

Proof For $\left.\tau \in \Pi\right|_{U}(k-1)$ we have that

$$
\left(\left(\ell \circ \iota_{X}\right) \cdot c\right)(\tau)=-\sum_{\sigma \succ \tau} c(\sigma) \ell\left(v_{\sigma \backslash \tau}\right)=-\ell\left(\sum_{\sigma \succ \tau} c(\sigma) v_{\sigma \backslash \tau}\right) .
$$

The statement follows from the fact that, for a vector $v \in N_{X}$, the condition $v=0$ is equivalent to $\ell(v)=0$ for every linear function $\ell$.

Proposition 3.19 Let $\Pi$ be a polyhedral complex on $X$ and $c$ a $k$-dimensional Minkowski weight on $\left.\Pi\right|_{U}$.

(1) If $V$ is an open subset of $U$, then $\left.c\right|_{V}$ is a $k$-dimensional Minkowski weight on $\left.\Pi\right|_{V}$.

(2) If $\Pi^{\prime}$ is a subdivision of $\Pi$, then $c_{\Pi^{\prime}}$ is a $k$-dimensional Minkowski weight on $\left.\Pi^{\prime}\right|_{U}$.

(3) If $f$ is a piecewise affine function on $U$ defined on $\Pi$, then $f \cdot c$ is a $(k-1)$-dimensional Minkowski weight on $\left.\Pi\right|_{U}$.

Proof The statement in (1) is direct from the definitions.

To prove (2), take a linear form $\ell$ on $N_{X}$ and set $h=\ell \circ \iota_{X}$ for short. Proposition 3.12 and Lemma 3.18 imply that

$$
h \cdot c_{\Pi^{\prime}}=(h \cdot c)_{\Pi^{\prime}}=0 .
$$

Since this holds for every $\ell$, Lemma 3.18 implies that $c_{\left.\Pi^{\prime}\right|_{U}}$ is a $k$-dimensional Minkowski weight on $\left.\Pi^{\prime}\right|_{U}$, proving the statement.

For (3), taking again a linear form $\ell$ on $N_{X}$ and setting $h=\ell \circ \iota_{X}$, we get from Proposition 3.12 and Lemma 3.18 that

$$
h \cdot(f \cdot c)=f \cdot(h \cdot c)=0 .
$$

Since this holds for every $\ell$, Lemma 3.18 again implies that $f \cdot c$ is a $(k-1)$-dimensional Minkowski weight on $\left.\Pi\right|_{U}$, as stated. 
Hence the operations of restriction to an open subset, pullback to a subdivision and product with piecewise affine functions induce the families of homomorphisms that in the notation of Proposition 3.19, write down, for each $k \in \mathbb{Z}_{\geq 0}$, as

$$
\begin{aligned}
& M_{k}\left(\left.\Pi\right|_{U}\right) \longrightarrow M_{k}\left(\left.\Pi\right|_{V}\right),\left.\quad c \longmapsto c\right|_{V}, \\
& M_{k}\left(\left.\Pi\right|_{U}\right) \longrightarrow M_{k}\left(\left.\Pi^{\prime}\right|_{U}\right), \quad c \longmapsto c_{\Pi^{\prime}}, \\
& \operatorname{PA}_{\Pi}(U) \times M_{k}\left(\left.\Pi\right|_{U}\right) \longrightarrow M_{k-1}\left(\left.\Pi\right|_{U}\right), \quad(f, c) \longmapsto f \cdot c .
\end{aligned}
$$

Definition 3.23 For $k \in \mathbb{Z}_{\geq 0}$, the space of Minkowski cycles on $U$ of dimension $k$ is defined as the direct limit

$$
Z_{k}(U)=\underset{\Pi}{\lim } M_{k}\left(\left.\Pi\right|_{U}\right)
$$

taken over the directed set of polyhedral complexes on $X$ ordered by subdivision, and where the map corresponding to each pair $\Pi^{\prime} \geq \Pi$ is the pullback homomorphism in (3.21).

Given a Minkowski weight $c \in M_{k}\left(\left.\Pi\right|_{U}\right)$, we denote by $[c] \in Z_{k}(U)$ the associated Minkowski cycle. Conversely, given a Minkowski cycle $\gamma \in Z_{k}(U)$ and a polyhedral complex $\Pi$ on $X$, we say that $\gamma$ is defined on $\Pi$ if there is $c \in M_{k}\left(\left.\Pi\right|_{U}\right)$ such that $\gamma=[c]$. The support of a Minkowski cycle $\gamma$ is the support of any Minkowski weight representing it.

The Minkowski cycle $\gamma$ is positive if it can be represented by a positive Minkowski weight on $\left.\Pi\right|_{U}$. We denote by $Z_{k}^{+}(U)$ the cone of positive Minkowski cycles on $U$ of dimension $k$.

The compatibility between the pull-back of Minkowski weights to subdivisions on the one hand and the restriction to open subsets and the product with piecewise affine functions on the other (Proposition 3.12) allows to define the corresponding operations for Minkowski cycles. Namely, from (3.20) and (3.22) we derive the families of homomorphisms given, for $k \in \mathbb{Z}_{\geq 0}$, by

$$
\begin{aligned}
& Z_{k}(U) \longrightarrow Z_{k}(V),\left.\quad \gamma \longmapsto \gamma\right|_{V}:=\left[\left.c\right|_{V}\right], \\
& \operatorname{PA}(U) \times Z_{k}(U) \longrightarrow Z_{k-1}(U), \quad(f, \gamma) \longmapsto f \cdot \gamma:=[f \cdot c]
\end{aligned}
$$

for any polyhedral complex $\Pi$ on $X$ and $c \in M_{k}\left(\left.\Pi\right|_{U}\right)$ with $\gamma=[c]$.

We next introduce the notions of balancing condition and balanced polyhedral space.

Definition 3.24 Suppose that the Euclidean polyhedral space $X$ has pure dimension $n$. A balancing condition on $U$ is an $n$-dimensional Minkowski cycle $\beta$ on $U$ that is represented by an $n$-dimensional Minkowski weight $b$ on $\left.\Pi\right|_{U}$ for a polyhedral complex $\Pi$ on $X$, and such that $b(\sigma)>0$ for all $\left.\sigma \in \Pi\right|_{U}(n)$. The pair $(U, \beta)$ is called a balanced open subset. When $U=X$, the pair $(X, \beta)$ is called a balanced polyhedral space. A polyhedral complex $\Pi$ on a balanced open subset $(U, \beta)$ is a polyhedral complex such that the balancing condition $\beta$ is defined on $\Pi$.

The Euclidean polyhedral space $X$ is balanceable if it admits a balancing condition on $X$. It is locally balanceable if there is an open covering $X=\bigcup_{i} U_{i}$ admitting a balancing condition on each $U_{i}$.

We will usually denote a balanced open subset by its underlying subset $U$ and, in this case, we will denote the corresponding balancing condition by $\beta_{U}$.

Example 3.25 For $n \in \mathbb{Z}_{\geq 0}$, the vector space $\mathbb{R}^{n}$ can be given a structure of a balanced polyhedral space by considering the polyhedral complex $\Pi$ consisting of the single polyhedron 
Fig. 1 A locally balanceable space

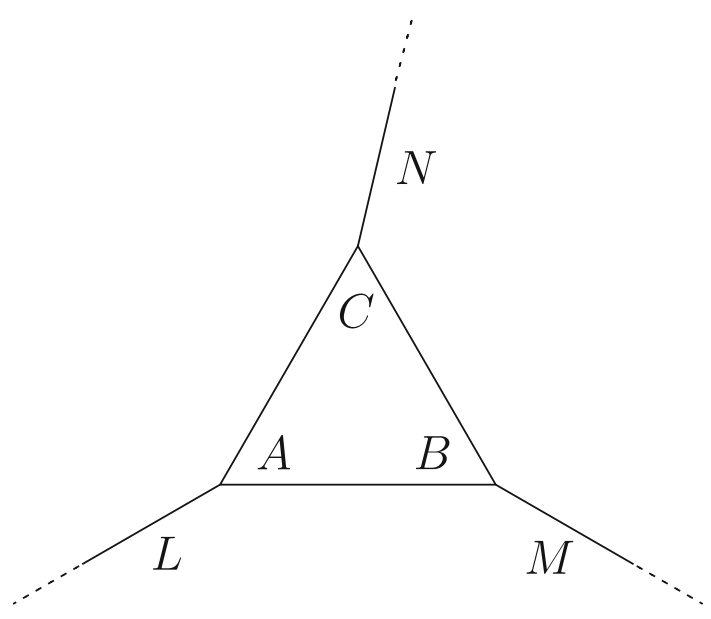

$\sigma=\mathbb{R}^{n}$ embedded in the Euclidean vector space $\mathbb{R}^{n+1}$ through the map $x \mapsto(x, 1)$, and the Minkowski weight defined by $\beta_{\mathbb{R}^{n}}(\sigma)=1$.

Remark 3.26 As in Example 3.25, in many situations the space $N$ will consist of the Euclidean space $\mathbb{R}^{n+1}$ and $H$ of the hyperplane $\left(x_{n+1}=1\right)$. In those situations we will only explicit the hyperplane $H$, whereas the space $N$ will be tacitly assumed.

Example 3.27 Let $X$ be a Euclidean polyhedral space, $U \subset X$ an open subset and $\gamma \in Z_{k}^{+}(U)$ a positive Minkowski cycle. Then the support $|\gamma|$ has an induced structure of Euclidean polyhedral space of pure dimension $k$ with a balancing condition on $|\gamma| \cap U$, given by the restriction of the cycle $\gamma$ to its support. In particular, if $U=X$ then $|\gamma|$ is balanced.

Example 3.28 Not every Euclidean polyhedral space is balanceable. For instance, the ray $\mathbb{R}_{\geq 0}$ with its standard structure of a Euclidean polyhedral space given by the polyhedra $\{0\}$ and $\mathbb{R}_{\geq 0}$, is not balanceable. Indeed, let $\Pi$ be a polyhedral complex on $\mathbb{R}_{\geq 0}$ and set $\tau=\{0\} \in \Pi(0)$. There is a unique polyhedron $\sigma \in \Pi(1)$ with $\tau \prec \sigma$ and, for any $c \in M_{1}(\Pi)$, the condition (3.4) boils down to

$$
c(\sigma) v_{\sigma \backslash \tau}=0 .
$$

Hence $c(\sigma)=0$ and so no balancing condition can be defined on $\Pi$.

More generally, any convex polyhedron $\Delta \subset \mathbb{R}^{n}$ of maximal dimension is a Euclidean polyhedral space with the structure induced from that of $\mathbb{R}^{n}$ in Example 3.25 but, unless $\Delta=\mathbb{R}^{n}$, it is not balanceable.

Example 3.29 Let $X$ be the Euclidean polyhedral space depicted in Fig. 1, where $A B C$ is an equilateral triangle, $L$ is the bisector of the angle opposed to $A$ and $M$ is the bisector of the angle opposed to $B$. Then $X$ is balanceable if and only if $N$ is the bisector of the angle opposed to $C$, while it is locally balanceable if an only if $N$ is contained in the interior of the angle opposed to $C$.

We end this section discussing the change of Euclidean structure.

Definition 3.30 Let $X^{\prime}$ be another Euclidean polyhedral space that has the same underlying quasi-embedded polyhedral space of $X$ and a possibly different Euclidean structure. Let 
$\Pi$ be a polyhedral complex on $X$ and $\Pi^{\prime}$ the corresponding polyhedral complex on $X^{\prime}$. For $k \in \mathbb{Z}_{\geq 0}$, the change of Euclidean structure on the open subset $U$ is the morphism $\varphi_{X^{\prime}, X}: W_{k}\left(\left.\Pi\right|_{U}\right) \rightarrow W_{k}\left(\left.\Pi^{\prime}\right|_{U}\right)$ given, for $c \in W_{k}\left(\left.\Pi\right|_{U}\right)$ and $\left.\sigma \in \Pi\right|_{U}(k)$, by

$$
\varphi_{X^{\prime}, X}(c)(\sigma)=\frac{\operatorname{vol}_{X^{\prime}}(\sigma)}{\operatorname{vol}_{X}(\sigma)} c(\sigma),
$$

where $\operatorname{vol}_{X^{\prime}}(\sigma) / \operatorname{vol}_{X}(\sigma)$ denotes the ratio between the Haar measures on the affine space $N_{\sigma}$ induced by the Euclidean structures of $X^{\prime}$ and $X$.

Remark 3.31 It follows directly form the definition that for three Euclidean structures $X, X^{\prime}$ and $X^{\prime \prime}$ on the same polyhedral space, the change of Euclidean structure maps induced by a polyhedral complex on $X$ verify that

$$
\varphi_{X^{\prime \prime}, X}=\varphi_{X^{\prime \prime}, X^{\prime}} \circ \varphi_{X^{\prime}, X}
$$

Proposition 3.32 With notation as in Definition 3.30, let $c \in M_{k}\left(\left.\Pi\right|_{U}\right)$ and $f \in \mathrm{PA}_{\Pi}(U)$. Then

$$
\varphi_{X^{\prime}, X}(c) \in M_{k}\left(\left.\Pi^{\prime}\right|_{U}\right) \text { and } \varphi_{X^{\prime}, X}(f \cdot c)=f \cdot \varphi_{X^{\prime}, X}(c) .
$$

Proof We denote with a prime the objects relative to $X^{\prime}$. For instance, for $\left.\tau \in \Pi\right|_{U}(k-1)$ and $\left.\sigma \in \Pi\right|_{U}(k)$ with $\tau \prec \sigma$, the symbol $v_{\sigma \backslash \tau}^{\prime}$ denotes the unit normal vector to $\tau$ in the direction of $\sigma$ for the Euclidean structure of $X^{\prime}$, while $v_{\sigma \backslash \tau}$ denotes that for $X$.

We decompose this vector as

$$
v_{\sigma \backslash \tau}^{\prime}=\left\langle v_{\sigma \backslash \tau}^{\prime}, v_{\sigma \backslash \tau}\right\rangle v_{\sigma \backslash \tau}+w_{\sigma \backslash \tau},
$$

where the scalar product is computed in $X$ and $w_{\sigma \backslash \tau}$ is a vector parallel to $\iota_{X, \tau}\left(H_{\tau}\right)$. The ratio between the Haar measures induced on the affine spaces $H_{\tau}$ and $H_{\sigma}$ are related by

$$
\frac{\operatorname{vol}_{X^{\prime}}(\tau)}{\operatorname{vol}_{X}(\tau)}=\left\langle v_{\sigma \backslash \tau}^{\prime}, v_{\sigma \backslash \tau}\right\rangle \frac{\operatorname{vol}_{X^{\prime}}(\sigma)}{\operatorname{vol}_{X}(\sigma)} .
$$

To prove that $\varphi_{X^{\prime}, X}(c)$ is a Minkowski weight we compute, for $\left.\tau \in \Pi\right|_{U}(k-1)$,

$$
\begin{aligned}
& \sum_{\sigma \succ \tau} \frac{\operatorname{vol}_{X^{\prime}}(\sigma)}{\operatorname{vol}_{X}(\sigma)} c(\sigma) v_{\sigma \backslash \tau}^{\prime}=\sum_{\sigma \succ \tau} \frac{\operatorname{vol}_{X^{\prime}}(\sigma)}{\operatorname{vol}_{X}(\sigma)} c(\sigma)\left(\left\langle v_{\sigma \backslash \tau}^{\prime}, v_{\sigma \backslash \tau}\right\rangle v_{\sigma \backslash \tau}+w_{\sigma \backslash \tau}\right) \\
& =\frac{\operatorname{vol}_{X^{\prime}}(\tau)}{\operatorname{vol}_{X}(\tau)}\left(\sum_{\sigma \succ \tau} c(\sigma) v_{\sigma \backslash \tau}\right)+w_{\tau}=w_{\tau}
\end{aligned}
$$

for some vector $w_{\tau}$ parallel to $\iota_{X, \tau}\left(H_{\tau}\right)$. Since $w_{\tau}$ is a linear combination of the vectors $v_{\sigma \backslash \tau}^{\prime}$, $\sigma \succ \tau$, and these vectors are orthogonal to the affine subspace $\iota_{X, \tau}\left(H_{\tau}\right)$ with respect to the Euclidean structure of $X^{\prime}$, we deduce that $w_{\tau}=0$, proving the first statement.

To prove the second statement, we compute, using the notation in (3.33) and the fact that $w_{\tau}=0$,

$$
\begin{aligned}
& \left(f \cdot \varphi_{X^{\prime}, X}(c)\right)(\tau)=-\sum_{\sigma \succ \tau} \frac{\operatorname{vol}_{X^{\prime}}(\sigma)}{\operatorname{vol}_{X}(\sigma)} c(\sigma) f_{\sigma}\left(v_{\sigma \backslash \tau}^{\prime}\right) \\
& =-\frac{\operatorname{vol}_{X^{\prime}}(\tau)}{\operatorname{vol}_{X}(\tau)}\left(\sum_{\sigma \succ \tau} c(\sigma) f_{\sigma}\left(v_{\sigma \backslash \tau}\right)\right)-f_{\tau}\left(w_{\tau}\right)=\varphi_{X^{\prime}, X}(f \cdot c)(\tau) .
\end{aligned}
$$


Thanks to this result, we can consider the change of Euclidean structure map from Definition 3.30 at the level of Minkowski weights and Minkowski cycles:

$$
\varphi_{X^{\prime}, X}: M_{k}\left(\left.\Pi\right|_{U}\right) \rightarrow M_{k}\left(\left.\Pi^{\prime}\right|_{U}\right) \quad \text { and } \quad \varphi_{X^{\prime}, X}: Z_{k}(U) \rightarrow Z_{k}\left(U^{\prime}\right) .
$$

They also satisfy the composition property of Remark 3.31 .

\section{Concave piecewise affine functions on polyhedral spaces}

In this section we introduce and discuss the different notions of concavity for a piecewise affine function on an open subset of a polyhedral space. We denote by $X$ a quasi-embedded polyhedral space and $U$ an open subset of it.

First we recall the definition of a concave function defined on a vector space and possibly taking infinite values.

Definition 4.1 A function $f: \mathbb{R}^{n} \rightarrow \mathbb{R} \cup\{ \pm \infty\}$ is concave if its hypograph

$$
\operatorname{hypo}(f)=\left\{(x, z) \in \mathbb{R}^{n} \times \mathbb{R} \mid z \leq f(x)\right\}
$$

is a convex subset of $\mathbb{R}^{n} \times \mathbb{R}$.

For a function $f: \mathbb{R}^{n} \rightarrow \mathbb{R} \cup\{-\infty\}$, being concave in the sense of Definition 4.1 is equivalent to the usual condition that, for all $x, x^{\prime} \in \mathbb{R}^{n}$ and $v_{1}, v_{2} \in \mathbb{R}_{\geq 0}$ with $v_{1}+v_{2}=1$,

$$
f\left(v_{1} x+v_{2} x^{\prime}\right) \geq v_{1} f(x)+v_{2} f\left(x^{\prime}\right) .
$$

However, this definition cannot be directly extended to functions on the open subset $U$, as we do not dispose of a notion of convexity for subsets of a polyhedral space. Instead, we can use the quasi-embedding to pullback the notion of concavity for functions on the vector space $N_{X}$.

Definition 4.2 Let $f$ be a piecewise affine function on $U$. Then $f$ is strongly concave if there is a concave function $f^{\prime}: H_{X} \rightarrow \mathbb{R} \cup\{ \pm \infty\}$ as in Definition 4.1, such that

$$
f=\left.\left(f^{\prime} \circ \iota_{X}\right)\right|_{U}
$$

The piecewise affine function $f$ is locally strongly concave if there is an open covering $U=\bigcup_{i} U_{i}$ such that $\left.f\right|_{U_{i}}$ is strongly concave for every $i$.

Similar definitions have been proposed in the context of tropical geometry, as in [11, Section 1.5]. However, neither of them is completely satisfactory. On the one hand, the notion of being strongly concave is not local as shown by the next example but, as we will see in Proposition 5.11, can be extended to a notion that is stable under the operation of taking the infimum. On the other hand, the notion of being locally strongly concave is local, but as also shown in the next example, cannot be extended to a notion that is stable under the operation of taking the infimum.

Example 4.3 Let $X$ be the 1-dimensional polyhedral space shown in Fig. 2 and consisting of the four polyhedra $\sigma_{i}=\mathbb{R}_{\geq 0} \times\{i\}, i=1, \ldots, 4$, glued together by the points $(0, i)$. Set $H=\mathbb{R}^{2}$ and let $\iota: X \rightarrow H$ be the quasi-embedding defined, for $x \in \mathbb{R}_{\geq 0}$ and $i \in\{1,2,3,4\}$, 
Fig. 2 A polyhedral complex

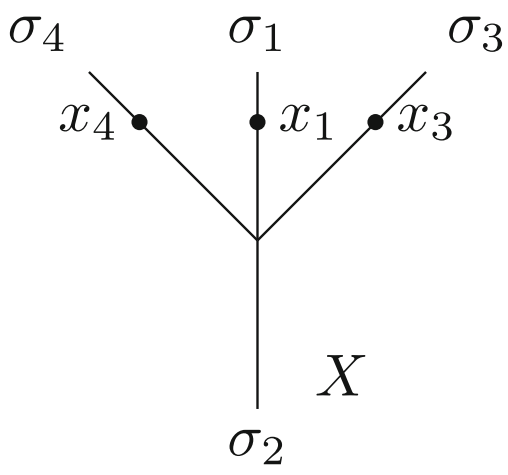

by

$$
\iota(x, i)= \begin{cases}(0, x) & \text { if } i=1 \\ (0,-x) & \text { if } i=2 \\ (x, x) & \text { if } i=3 \\ (-x, x) & \text { if } i=4\end{cases}
$$

For each integer $n \geq 2$ consider the piecewise affine function $f_{n}: X \rightarrow \mathbb{R}$ given by

$$
f_{n}(x, i)= \begin{cases}\min \left(0, \frac{1}{n}-x\right) & \text { if } i=1, \\ 0 & \text { otherwise. }\end{cases}
$$

It is not strongly concave because for the points $x_{1}=\iota(1,1), x_{3}=\iota(1,3)$ and $x_{4}=\iota(1,4)$ shown in Fig. 2 we have that $\iota\left(x_{1}\right)=\frac{1}{2} x_{3}+\frac{1}{2} x_{4}$ and

$$
f_{n}(1,1)=\frac{1}{n}-1<0=\frac{1}{2} f_{n}(1,3)+\frac{1}{2} f_{n}(1,4) .
$$

Hence $f_{n}$ is not the pullback to $X$ of a concave function on $H$. On the other hand, this piecewise affine function is locally strongly concave, as it can be seen by considering the covering $X=U_{n} \cup V_{n}$ with

$$
U_{n}=\left\{(x, i) \mid i \neq 1 \text { or } x<\frac{2}{3 n}\right\} \quad \text { and } \quad V_{n}=\left\{(x, i) \mid i=1 \text { and } x>\frac{1}{3 n}\right\} .
$$

This shows that the notions of being strongly concave and of being locally strongly concave do not agree, and the notion of being strongly concave is not local.

Moreover, consider the piecewise affine function $f: X \rightarrow \mathbb{R}$ defined by

$$
f(x, i)= \begin{cases}-x & \text { if } i=1, \\ 0 & \text { otherwise }\end{cases}
$$

It is the infimum of the family $\left\{f_{n}\right\}_{n \geq 2}$, but it is not locally strongly concave. Indeed, for any $\varepsilon>0$ we have that $\iota(\varepsilon, 1)=\frac{1}{2} \iota(\varepsilon, 3)+\frac{1}{2} \iota(\varepsilon, 4)$ and

$$
f(\varepsilon, 1)=-\varepsilon<0=\frac{1}{2} f(\varepsilon, 3)+\frac{1}{2} f(\varepsilon, 4),
$$

and so the restriction of $f$ to any open neighborhood of the point $(0,1)$ is not the pullback of a concave function on $H$. Thus the notion of being locally strongly concave is not closed under the operation of taking the infimum. 
In Definition 4.2, the concave function $f^{\prime}$ on $H_{X}$ might in principle take the values $\pm \infty$, even though the piecewise affine function $f$ on $U$ takes only values in $\mathbb{R}$. The next example shows that including these infinite values does make a difference in the notion of strong concavity, with respect to a definition where these values for $f^{\prime}$ are not allowed.

Example 4.5 Let $X$ be the 1-dimensional polyhedral space consisting of the union of the two real lines $\mathbb{R} \times\{0\}$ and $\mathbb{R} \times\{1\}$ of $H=\mathbb{R}^{2}$. Consider the piecewise affine function $f: X \rightarrow \mathbb{R} \cup\{ \pm \infty\}$ defined, for $x \in \mathbb{R}$, by $f(x, 0)=x$ and $f(x, 1)=-x$, and let $f^{\prime}: \mathbb{R}^{2} \rightarrow \mathbb{R}$ be the function defined, for $(x, y) \in \mathbb{R}^{2}$, by

$$
f^{\prime}(x, y)= \begin{cases}+\infty & \text { if } 0<y<1, \\ x & \text { if } y=0, \\ -x & \text { if } y=1, \\ -\infty & \text { if } y<0 \text { or } y>1 .\end{cases}
$$

It is concave according to Definition 4.1 and since $f=f^{\prime} \circ \iota$, we deduce that $f$ is strongly concave in the sense of Definition 4.2. Moreover, any concave function $f^{\prime \prime}: \mathbb{R}^{2} \rightarrow \mathbb{R} \cup\{ \pm \infty\}$ with $f=f^{\prime \prime} \circ \iota$ should satisfy that

$$
f^{\prime \prime}\left(0, \frac{1}{2}\right) \geq \frac{1}{2} f(x, 0)+\frac{1}{2} f(-x, 1)=x
$$

for all $x \in \mathbb{R}$. Hence, such a concave function necessarily takes the value $+\infty$ at the point $\left(0, \frac{1}{2}\right)$.

In view of the issues concerning these notions of concavity, we propose two other ones based on the idea of preserving the positivity of Minkowski cycles. For the rest of this section, we assume that $X$ is a Euclidean polyhedral space and that $U$ is an open subset of it.

Definition 4.6 Let $f$ be a piecewise affine function on $U$.

(1) The piecewise affine function $f$ is concave if for every positive Minkowski cycle $\gamma$ on an open subset $V$ of $U$, the product $f \cdot \gamma$ is also a positive Minkowski cycle on $V$.

(2) When $U$ is balanced with balancing condition $\beta_{U}$, then $f$ is weakly concave if the product $f \cdot \beta_{U}$ is a positive Minkowski cycle on $U$.

The sets of strongly concave, of concave, and of weakly concave piecewise affine functions on $U$ are respectively denoted by

\section{$\operatorname{SCPA}(U), \quad \operatorname{CPA}(U), \quad \operatorname{WCPA}(U)$.}

These are cones in $\mathrm{PA}(U)$. Similarly, for a polyhedral complex $\Pi$ on $X$, the cones of strongly concave, of concave, and of weakly concave piecewise affine functions on $U$ that are defined on $\Pi$ are respectively denoted by

\section{$\operatorname{SCPA}_{\Pi}(U), \quad \mathrm{CPA}_{\Pi}(U), \quad \mathrm{WCPA}_{\Pi}(U)$.}

When we want to stress the dependency of the class of weakly concave piecewise affine functions on the balancing condition, we denote its corresponding spaces by $\operatorname{WCPA}\left(U, \beta_{U}\right)$ and $\mathrm{WCPA}_{\Pi}\left(U, \beta_{U}\right)$.

Clearly, the notion of strongly concave piecewise affine function does not depend on the choice of the Euclidean structure of $X$. The next result shows the dependency of the other notions of concavity with respect to changes on the Euclidean structure. 
Proposition 4.7 Let $X^{\prime}$ be a Euclidean polyhedral space with the same quasi-embedded polyhedral space of $X$, let $U^{\prime}$ be the open subset of $X^{\prime}$ corresponding to $U$ and $\varphi_{X^{\prime}, X}: Z_{k}(U) \rightarrow$ $Z_{k}\left(U^{\prime}\right), k \in \mathbb{Z}_{\geq 0}$, the change of Euclidean structure maps as in (3.34). Then

$$
\operatorname{CPA}(U)=\operatorname{CPA}\left(U^{\prime}\right) \text { and } \operatorname{WCPA}\left(U, \beta_{U}\right)=\operatorname{WCPA}\left(U^{\prime}, \varphi_{X^{\prime}, X}\left(\beta_{U}\right)\right) .
$$

Proof This is a direct consequence of Proposition 3.32.

We have seen in Example 4.3 that, for a piecewise affine function on an open subset of a polyhedral space, the notion of being strongly concave is not local. By contrast, the notions of being concave and of being weakly concave are local.

Proposition 4.8 Let $f$ be a piecewise affine function on $U$, and $U=\bigcup_{i} U_{i}$ an open covering.

(1) $f$ is concave if and only if $\left.f\right|_{U_{i}}$ is concave for all $i$.

(2) When $U$ is balanced, $f$ is weakly concave if and only if $\left.f\right|_{U_{i}}$ is weakly concave for all $i$.

Proof This follows directly from the definitions and the compatibility between the restriction to open subsets and the product of piecewise affine functions with Minkowski weights (Proposition 3.12).

The next result motivates our terminology for the different notions of concavity.

Proposition 4.9 Let $f$ be a piecewise affine function on $U$.

(1) If $f$ is locally strongly concave, then $f$ is concave.

(2) If $U$ is balanced and $f$ is concave, then $f$ is weakly concave.

Proof To prove (1), let $\Pi$ be a polyhedral complex on $X$ where $f$ is defined, $V \subset U$ an open subset, $k \in \mathbb{Z}_{\geq 0}$, and $c \in M_{k}^{+}\left(\left.\Pi\right|_{V}\right)$. Let $\left.\tau \in \Pi\right|_{V}(k-1)$ and set

$$
S=\sum_{\substack{\sigma \in \prod_{\sigma>\tau}(k) \\ \sigma>\tau}} c(\sigma)
$$

If $S=0$ then $c(\sigma)=0$ for all $\left.\sigma \in \Pi\right|_{V}(k)$ with $\sigma \succ \tau$ because $c$ is positive. Therefore $(f \cdot c)(\tau)=0$ in this case.

Else $S>0$, and pick then $x \in \operatorname{relint}(\tau) \cap V$. Since $f$ is locally strongly concave, we can choose an open neighborhood $V^{\prime}$ in $V$ of the point $x$, a convex subset $C$ of $N_{X}$ and a concave function $f^{\prime}: C \rightarrow \mathbb{R}$ such that

$$
\left.f\right|_{V^{\prime}}=f^{\prime} \circ \iota_{X}
$$

Choose also $\varepsilon>0$ so that $\iota_{X}(x)+\varepsilon v_{\sigma \backslash \tau} \in \iota_{X}\left(\sigma \cap V^{\prime}\right)$ for all $\left.\sigma \in \Pi\right|_{V}(k)$ with $\sigma \succ \tau$. By (4.10) we have that $\sum_{\sigma \succ \tau} \frac{c(\sigma)}{S}=1$ and, since $c$ is a Minkowski weight, we also have that $\sum_{\sigma \succ \tau} c(\sigma) v_{\sigma \backslash \tau}=0$. Therefore, the concavity of $f^{\prime}$ implies that

$$
f^{\prime}\left(\iota_{X}(x)\right) \geq \sum_{\sigma \succ \tau} \frac{c(\sigma)}{S} f^{\prime}\left(\iota_{X}(x)+\varepsilon v_{\sigma \backslash \tau}\right) .
$$

From the formula in (3.11) and the fact that $f$ is affine on each polyhedron $\left.\sigma \in \Pi\right|_{V}$ we get that

$$
(f \cdot c)(\tau)=\frac{S}{\varepsilon}\left(f^{\prime}\left(\iota_{X}(x)\right)-\sum_{\sigma \succ \tau} \frac{c(\sigma)}{S} f^{\prime}\left(\iota_{X}(x)+\varepsilon v_{\sigma \backslash \tau}\right)\right),
$$

and so (4.11) implies that $(f \cdot c)(\tau) \geq 0$. Hence $f \cdot c$ is positive, and thus $f$ is concave.

The statement in (2) is immediate from the definitions, because the balancing condition $\beta_{U}$ is a positive Minkowski cycle. 
The next result shows that, for a given piecewise affine function, the condition of being concave can be checked in any polyhedral complex where it is defined.

Proposition 4.12 Let $f$ be a piecewise affine function on $U$ defined on a polyhedral complex П. The following conditions are equivalent:

(1) $f$ is concave,

(2) for every open subset $V \subset U$, every $k \in \mathbb{Z}_{\geq 0}$ and every $c \in M_{k}^{+}\left(\left.\Pi\right|_{V}\right)$, we have that $f \cdot c \in M_{k-1}^{+}\left(\left.\Pi\right|_{V}\right)$.

Proof Suppose that $f$ is concave and let $c \in M_{k}^{+}\left(\left.\Pi\right|_{V}\right)$. Since $[f \cdot c]=f \cdot[c]$ is a positive Minkowski cycle, we deduce that $f \cdot c$ is a positive Minkowski weight, and so $f$ satisfies the condition (2).

To prove the converse, suppose that $f$ satisfies the condition (2) and choose an open subset $V \subset U, k \in \mathbb{Z}_{\geq 0}$ and $\gamma \in Z_{k}^{+}(V)$. Let $\Pi^{\prime}$ be a subdivision of $\Pi$ and $c^{\prime} \in M_{k}^{+}\left(\left.\Pi^{\prime}\right|_{V}\right)$ a positive Minkowski weight representing $\gamma$.

Let $\left.\tau \in \Pi^{\prime}\right|_{V}(k-1)$ and set $\mu$ for the minimal polyhedron of $\left.\Pi\right|_{V}$ containing $\tau$. For each $\left.\sigma \in \Pi^{\prime}\right|_{V}(k)$ with $\sigma>\tau$, let $\lambda(\sigma)$ be the minimal polyhedron of $\left.\Pi\right|_{V}$ containing $\sigma$. We decompose the unit normal vector to $\tau$ in the direction of $\sigma$ as

$$
v_{\sigma \backslash \tau}=v_{\sigma, \mu}+v_{\sigma, \mu^{\perp}}
$$

with $v_{\sigma, \mu}$ parallel to the affine subspace $\iota_{X}\left(H_{\mu}\right)$ and $v_{\sigma, \mu^{\perp}}$ orthogonal to it. Then

$$
0=\sum_{\sigma \succ \tau} c^{\prime}(\sigma) v_{\sigma \backslash \tau}=\sum_{\sigma \succ \tau} c^{\prime}(\sigma) v_{\sigma, \mu}+\sum_{\sigma \succ \tau} c^{\prime}(\sigma) v_{\sigma, \mu^{\perp}}
$$

Since this decomposition is orthogonal, we deduce that

$$
\sum_{\sigma \succ \tau} c^{\prime}(\sigma) v_{\sigma, \mu}=\sum_{\sigma \succ \tau} c^{\prime}(\sigma) v_{\sigma, \mu^{\perp}}=0
$$

Put $l=\operatorname{dim}(\mu)$, and for each $\sigma$ as above write

$$
v_{\sigma, \mu^{\perp}}=\sum_{\mu \prec \nu \prec \lambda(\sigma)} a_{\sigma, \nu} v_{\nu \backslash \mu}
$$

with $a_{\sigma, v} \in \mathbb{R}_{\geq 0}$ and where the sum goes over the polyhedra $\left.v \in \Pi\right|_{V}(l+1)$ with $\mu \prec v \prec$ $\lambda(\sigma)$. Such a decomposition does exist because $\mu \prec \lambda(\sigma)$, and the cone of vectors that are orthogonal to $\mu$ and point towards $\lambda(\sigma)$ contains $v_{\sigma, \mu^{\perp}}$ and is generated by the vectors $v_{\nu \backslash \mu}$ for $\left.v \in \Pi\right|_{V}(l+1)$ with $\mu \prec v \prec \lambda(\sigma)$.

Let $W$ be an open subset of $V$ such that $\left.\Pi^{\prime}\right|_{W}$ consists only of the polyhedra having $\tau$ as a face. We then define a positive Minkowski weight $c \in M_{l+1}^{+}\left(\left.\Pi\right|_{W}\right)$ by setting, for each $\left.v \in \Pi\right|_{W}(l+1)$,

$$
c(\nu)=\sum_{\substack{\sigma \succ \tau \\ \lambda(\sigma) \succ v}} a_{\sigma, \nu} c^{\prime}(\sigma),
$$

the sum being over the polyhedra $\left.\sigma \in \Pi^{\prime}\right|_{V}(k)$ with $\sigma \succ \tau$ and $\lambda(\sigma) \succ v$. Since $\left.\Pi\right|_{W}(l)=$ $\{\mu\}$, to see that the formula in (4.16) defines a positive Minkowski weight we only need to 
check the balancing condition at this polyhedron:

$$
\begin{aligned}
& \sum_{\nu \succ \mu} c(\nu) v_{\nu \backslash \mu}=\sum_{\nu \succ \mu} \sum_{\substack{\sigma \succ \tau \\
\lambda(\sigma) \succ \nu}} a_{\sigma, \nu} c^{\prime}(\sigma) v_{\nu \backslash \mu} \\
& =\sum_{\sigma \succ \tau} c^{\prime}(\sigma)\left(\sum_{\mu \prec \nu \prec \lambda(\sigma)} a_{\sigma, \nu} v_{\nu \backslash \mu}\right)=\sum_{\sigma \succ \tau} c^{\prime}(\sigma) v_{\sigma, \mu^{\perp}}=0,
\end{aligned}
$$

where the sums are taken over the polyhedra $\left.v \in \Pi\right|_{W}(l+1)$ and $\left.\sigma \in \Pi^{\prime}\right|_{W}(k)$ subject to the conditions stated therein. Moreover, the product $\left(f \cdot c^{\prime}\right)(\tau)$ coincides with $(f \cdot c)(\mu)$ :

$$
\begin{aligned}
\left(f \cdot c^{\prime}\right)(\tau) & =-\sum_{\sigma \succ \tau} c^{\prime}(\sigma) f_{\sigma}\left(v_{\sigma \backslash \tau}\right) \\
& =-\sum_{\sigma \succ \tau} c^{\prime}(\sigma) f_{\lambda(\sigma)}\left(v_{\sigma, \mu}+v_{\sigma, \mu^{\perp}}\right) \\
& =-\sum_{\sigma \succ \tau} c^{\prime}(\sigma) f_{\mu}\left(v_{\sigma, \mu}\right)-\sum_{\sigma \succ \tau} \sum_{\mu \prec \nu \prec \lambda(\sigma)} c^{\prime}(\sigma) a_{\sigma, \nu} f_{\lambda(\sigma)}\left(v_{\nu \backslash \mu}\right) \\
& =-f_{\mu}(0)-\sum_{\mu \prec \nu} c(\nu) f_{\nu}\left(v_{\nu \backslash \mu}\right) \\
& =(f \cdot c)(\mu),
\end{aligned}
$$

where the indexes $v$ and $\sigma$ denote polyhedra in $\left.\Pi\right|_{W}(l+1)$ and in $\left.\Pi^{\prime}\right|_{W}(k)$, respectively: the first equality is the definition of the product, the second follows from the decomposition in (4.13) and the fact that $f_{\sigma}$ and $f_{\lambda(\sigma)}$ coincide on $\iota_{X}\left(N_{\sigma}\right)$, the third from the decomposition in (4.15) and the fact that $f_{\lambda(\sigma)}$ and $f_{\mu}$ coincide on $\iota_{X}\left(N_{\mu}\right)$, the fourth from the first equation in (4.14), the definition of the Minkowski weight $c$ and the fact that $f_{\lambda(\sigma)}$ and $f_{\nu}$ coincide on $\iota_{X}\left(N_{v}\right)$, and the fifth from the definition of the product.

Since $c$ is positive, by hypothesis $(f \cdot c)(\mu) \geq 0$. Hence $\left(f \cdot c^{\prime}\right)(\tau) \geq 0$ and since $\left.\tau \in \Pi^{\prime}\right|_{V}(k-1)$ is arbitrary, we deduce that $f \cdot c^{\prime}$ is positive and so

$$
f \cdot \gamma=f \cdot\left[c^{\prime}\right]=\left[f \cdot c^{\prime}\right]
$$

is also positive. Varying $V, k$ and $\gamma$, we conclude that $f$ is concave.

The next three examples show that the proposed classes of concave piecewise affine functions are different.

Example 4.17 Let $X$ be the 1-dimensional polyhedral space made of the three polyhedra $\sigma_{i}=\mathbb{R}_{\geq 0} \times\{i\}, i=1,2,3$, glued together by the points $(0, i)$. Let $H=\mathbb{R}$ with its standard Euclidean structure and let $\iota: X \rightarrow H$ be the quasi-embedding defined, for $(x, i) \in X$, by

$$
\iota(x, i)= \begin{cases}-x & \text { if } i=1, \\ x & \text { if } i=2,3 .\end{cases}
$$

Consider the polyhedral complex $\Pi$ on $X$ made of the three rays $\sigma_{i}$ and the origin $\tau=(0,1)$ and, for $\gamma_{i} \in \mathbb{R}_{>0}, i=2,3$, consider the balancing condition $b \in M_{1}(\Pi)$ given by

$$
b\left(\sigma_{1}\right)=\gamma_{2}+\gamma_{3}, \quad b\left(\sigma_{2}\right)=\gamma_{2} \quad \text { and } \quad b\left(\sigma_{3}\right)=\gamma_{3} .
$$

A piecewise affine function $f: X \rightarrow \mathbb{R}$ defined on $\Pi$ is of the form $f(x, i)=a_{i} x$ with $a_{i} \in \mathbb{R}$. Then 
(1) $f$ is strongly concave if and only if $-a_{1} \geq a_{2}=a_{3}$,

(2) $f$ is concave if and only if $-a_{1} \geq a_{2}$ and $-a_{1} \geq a_{3}$,

(3) $f$ is weakly concave if and only if $-\left(\gamma_{2}+\gamma_{3}\right) a_{1} \geq \gamma_{2} a_{2}+\gamma_{3} a_{3}$.

Hence these classes of concave piecewise affine functions are different, and the notion of being weakly concave depends on the choice of the balancing condition.

The quasi-embedding in the previous example is not globally injective. In the next examples we will see that even when the quasi-embedding is a injective, the three notions of concavity can differ.

Example 4.18 Let $X$ be the 1-dimensional balanced polyhedral space in Example 4.3. Let $\Pi$ the polyhedral complex on $X$ made of the four rays $\sigma_{i}=\mathbb{R}_{\geq 0} \times\{i\}, i=1, \ldots, 4$, and their origin $\tau=(0,1)$, and $U$ an open neighborhood of this point.

The cone of positive Minkowski weights on $\left.\Pi\right|_{U}$ of dimension 1 is generated by the weights $c_{1}$ and $c_{2}$ given by

$$
\begin{aligned}
& c_{1}\left(\sigma_{1}\right)=1, \quad c_{1}\left(\sigma_{2}\right)=1, \quad c_{1}\left(\sigma_{3}\right)=0, \quad c_{1}\left(\sigma_{4}\right)=0, \\
& c_{2}\left(\sigma_{1}\right)=0, \quad c_{2}\left(\sigma_{2}\right)=\sqrt{2}, \quad c_{1}\left(\sigma_{3}\right)=1, \quad c_{1}\left(\sigma_{4}\right)=1 .
\end{aligned}
$$

The piecewise affine function $f: X \rightarrow \mathbb{R}$ given by the formula (4.4) is defined on $\Pi$ and we have that

$$
\left(f \cdot c_{1}\right)(\tau)=1 \quad \text { and } \quad\left(f \cdot c_{2}\right)(\tau)=0 .
$$

By Proposition 4.12 it is concave although, as it was explained in Example 4.3, it is not strongly concave.

In the next example we construct a weakly concave piecewise affine function that multiplied twice with the balancing condition gives a negative weight. In particular, such a piecewise affine function is not concave.

Example 4.19 Let $e_{i}, i=1, \ldots, 5$, be five linearly independent vectors in a vector space $M$ and consider the polyhedral space $X$ in $M$ made of the nine 2-dimensional cones $\sigma_{i}$, $i=1, \ldots, 9$, respectively generated by the pairs $\left\{e_{1}, e_{3}\right\},\left\{e_{1}, e_{4}\right\},\left\{e_{1}, e_{5}\right\},\left\{e_{2}, e_{3}\right\},\left\{e_{2}, e_{4}\right\}$, $\left\{e_{2}, e_{5}\right\},\left\{e_{3}, e_{4}\right\},\left\{e_{3}, e_{5}\right\}$ and $\left\{e_{4}, e_{5}\right\}$. Let $\iota: X \rightarrow H=\mathbb{R}^{3}$ be the quasi-embedding given by

$$
\begin{array}{ll}
\iota\left(e_{1}\right)=(0,0,1), \quad \iota\left(e_{2}\right)=(0,0,-1), \\
\iota\left(e_{3}\right)=(1,0,0), \quad \iota\left(e_{4}\right)=(0,1,0), \quad \iota\left(e_{5}\right)=(-1,-1,0) .
\end{array}
$$

Let $\Pi$ be the polyhedral complex on $X$ made of the origin of $M$, the five rays $\tau_{i}=\mathbb{R}_{\geq 0} e_{i}$, $i=1, \ldots, 5$, and the nine cones $\sigma_{i}, i=1, \ldots, 9$. Let $b \in M_{2}(\Pi)$ be the balancing condition on $\Pi$ given by

$$
b\left(\sigma_{i}\right)= \begin{cases}\sqrt{2} & \text { if } i=3,6 \\ 1 & \text { otherwise }\end{cases}
$$

Hence $X$ is balanced and its quasi-embedding is globally injective.

Let $f: X \rightarrow \mathbb{R}$ be the piecewise affine function defined on $\Pi$ given by the values

$$
f\left(e_{1}\right)=1, \quad f\left(e_{2}\right)=0, \quad f\left(e_{3}\right)=-1, \quad f\left(e_{4}\right)=0, \quad f\left(e_{5}\right)=0 .
$$


We have that

$$
(f \cdot b)\left(\tau_{1}\right)=1,(f \cdot b)\left(\tau_{2}\right)=1,(f \cdot b)\left(\tau_{3}\right)=0,(f \cdot b)\left(\tau_{4}\right)=0,(f \cdot b)\left(\tau_{5}\right)=0 .
$$

Hence the 1-dimensional Minkowski weight $f \cdot b$ is positive, and so $f$ is weakly concave. On the other hand $(f \cdot(f \cdot b))(\{0\})=-1$, and therefore $f$ is not concave.

We next show that weakly concave piecewise affine functions preserve the positivity of the Minkowski weights given by products of concave piecewise affine functions with the balancing condition. By contrast, the product of several weakly concave piecewise affine functions with the balancing condition is not necessarily positive, as already shown in Example 4.19.

Definition 4.20 Suppose that the open subset $U \subset X$ is balanced with balancing condition $\beta_{U}$. Then a Minkowski cycle $\gamma$ on $U$ of dimension $k$ is $\beta_{U}$-positive if there are positive real numbers $\alpha_{j}, j=1, \ldots, s$ and concave piecewise affine functions $f_{1, j}, \ldots, f_{n-k, j}$ on $U$, $j=1, \ldots, s$, such that

$$
\gamma=\sum_{j=1}^{s} \alpha_{j} f_{1, j} \cdots f_{n-k, j} \cdot \beta_{U} .
$$

A Minkowski weight on $U$ is $\beta_{U}$-positive if it represents a $\beta_{U}$-positive Minkowski cycle.

Proposition 4.21 Suppose that $U \subset X$ is balanced, and let $f$ be a weakly concave piecewise affine function and $\gamma$ a $\beta_{U}$-positive Minkowski cycle on $U$. Then $f \cdot \gamma$ is a $\beta_{U}$-positive Minkowski cycle on $U$.

Proof It is enough prove the statement for Minkowski cycles of the form

$$
\gamma=f_{1} \cdots f_{n-k} \cdot \beta_{U} \in Z_{k}(U)
$$

with $0 \leq k \leq n$ and $f_{i} \in \mathrm{CPA}(U), i=1, \ldots, n-k$. By Proposition 3.15, we have that $f \cdot \gamma=f_{1} \cdots f_{n-k} \cdot f \cdot \beta_{U}$ and the definitions of concave and weakly concave piecewise affine functions imply that this product is positive, as stated.

Proposition 4.22 For a piecewise affine function on a convex open subset $U$ of $\mathbb{R}^{n}$ with the structure of balanced polyhedral complex of Example 3.25, each of the conditions of being weakly concave, concave, locally strongly concave and strongly concave is equivalent to being concave in the usual sense.

Proof Let $f$ be a piecewise affine function on $U$. If $f$ is concave in the usual sense, then it is also locally strongly concave and, by Proposition 4.9, it also satisfies the other notions of concavity.

For the converse, it is enough to show that the notion of being weakly concave implies that of being concave in the usual sense. Let $\Pi$ be a polyhedral complex on $\mathbb{R}^{n}$ with the induced balancing condition $b \in M_{n}^{+}\left(\left.\Pi\right|_{U}\right)$, and let $f \in \mathrm{WCPA}_{\Pi}(U)$.

For each $\left.\tau \in \Pi\right|_{U}(n-1)$ denote by $\sigma,\left.\sigma^{\prime} \in \Pi\right|_{U}(n)$ the two polyhedra containing $\tau$ as a facet. Since $f$ is weakly concave,

$$
(f \cdot b)(\tau)=-f_{\sigma}\left(v_{\sigma \backslash \tau}\right)-f_{\sigma^{\prime}}\left(v_{\sigma^{\prime} \backslash \tau}\right) \geq 0 .
$$

The linear functions $f_{\sigma}$ and $f_{\sigma^{\prime}}$ coincide on $\iota_{X, \tau}\left(N_{\tau}\right)$ and $v_{\sigma \backslash \tau}=-v_{\sigma^{\prime} \backslash \tau}$, and so the inequality in (4.23) implies that the restriction of $f$ to any convex subset of the union $\sigma \cup \sigma^{\prime}$ is concave in the usual sense. 
Now let $x, x^{\prime} \in U$ be two different points such that the segment $\overline{x x^{\prime}}$ does not intersect the $(n-2)$-dimensional skeleton of $\left.\Pi\right|_{U}$. Then every point $y \in \overline{x x^{\prime}}$ has a convex neighborhood that is contained in a subset of the form $\sigma \cup \sigma^{\prime}$ as above, and the restriction of $f$ to such a neighborhood is concave in the usual sense. Since this condition is local, we deduce that $f$ is concave on the segment $\overline{x x^{\prime}}$.

Hence, $f$ is concave on each segment $\overline{x x^{\prime}}$ for the pairs $\left(x, x^{\prime}\right)$ in a dense open subset of $U \times U$. Since $f$ is continuous (Remark 3.6), this property extends to every segment in $U$ and so $f$ is concave therein, as stated.

Corollary 4.24 Let X be a Euclidean polyhedral space of pure dimension, $U$ a balanced open subset of $X$, and $f$ a weakly concave piecewise affine function on $U$. Let $\Pi$ be a polyhedral complex on $X$ and $\sigma \in \Pi$ a polyhedron contained in $U$. Then the restriction $\left.f\right|_{\sigma}$ is concave in the usual sense.

Proof We can assume without loss of generality that $\sigma$ is of maximal dimension. The balancing condition on $U$ induces a balancing condition on the interior of $\sigma$ that is a positive multiple of the standard balancing condition of a polyhedron of $\mathbb{R}^{n}$. By Proposition 4.22 the restriction of $f$ to the interior of $\sigma$ is concave in the usual sense. Since $f$ is continuous, it is also concave on the whole of $\sigma$.

Definition 4.25 Let $\Pi$ be a polyhedral complex on the Euclidean polyhedral space $X$. A piecewise affine function $f$ on the open subset $U$ is strictly concave on $\left.\Pi\right|_{U}$ if it is defined on $\Pi$ and for every integer $k$, every $\left.\tau \in \Pi\right|_{U}(k)$, every open subset $V \subset U$ with $V \cap \tau \neq \emptyset$ and every $c \in M_{k+1}^{+}\left(\left.\Pi\right|_{V}\right)$ with $c(\sigma)>0$ for some $\left.\sigma \in \Pi\right|_{V}(k+1)$ with $\sigma \succ \tau$, we have that

$$
(f \cdot c)(\tau)>0 .
$$

The polyhedral complex $\Pi$ is regular on $U$ if there is a piecewise affine function $f$ on $U$ that is strictly concave on $\left.\Pi\right|_{U}$.

Remark 4.27 The notion of strictly concave introduced in definition 4.25 is a direct generalization of the notion of strictly convex function on a fan used in toric geometry, see for instance [7, page 68]. But take into account that what is called convex in loc. cit., is called concave in the context of convex analysis.

For a convex polyhedron $\Delta$ in a Euclidean vector space, a concave piecewise affine function $f$ on it induces a polyhedral complex on $\Delta$, denoted by

$$
\Pi(f),
$$

given by its affinity loci or equivalently, by the projection in the convex polyhedron of the faces of the upper envelope of the hypograph of $f$. In more explicit terms, a subset $\sigma \subset \Delta$ is an element of $\Pi(f)$ if and only if there is an affine function $\ell: \Delta \rightarrow \mathbb{R}$ such that

$$
f(x)=\ell(x) \text { for } x \in \sigma \quad \text { and } \quad f(x)<\ell(x) \text { for } x \in \Delta \backslash \sigma .
$$

Indeed, this is the minimal polyhedral complex on $\Delta$ where $f$ is defined.

Classically, a polyhedral complex on a convex polyhedron is called regular if it is of the form $\Pi(f)$ for a concave piecewise affine function on it, see for instance [6, Definition 2.2.10]. The next result shows that Definition 4.25 is a generalization of this notion to arbitrary Euclidean polyhedral complexes. 
Proposition 4.30 Let $\Delta$ be a full dimensional polyhedron of $\mathbb{R}^{n}$ with the induced structure of Euclidean polyhedral space and set $U=\operatorname{relint}(\Delta)$. Let $\Pi$ be a polyhedral complex on $\Delta$ and $f \in \mathrm{PA}_{\Pi}(\Delta)$. Then $f$ is strictly concave on $\left.\Pi\right|_{U}$ if and only if it is concave in the usual sense and $\Pi=\Pi(f)$.

In particular, $\Pi$ is regular on $U$ in the sense of Definition 4.25 if and only if there is a concave piecewise affine function $f$ on $\Delta$ such that $\Pi=\Pi(f)$.

Proof First assume that $f$ is strictly concave on $\left.\Pi\right|_{U}$, and let $b \in M_{n}\left(\left.\Pi\right|_{U}\right)$ be the balancing condition on $\left.\Pi\right|_{U}$ induced from the standard one of $\mathbb{R}^{n}$, given in Example 3.25. Since $b(\sigma)>$ 0 for all $\left.\sigma \in \Pi\right|_{U}(n)$, the condition (4.26) implies that $f$ is weakly concave on $U$. By Proposition 4.22, it is concave on $U$ in the usual sense and by continuity, it is also concave on the whole of $\Delta$. Since $f$ is defined on $\Pi$, we have that $\Pi(f) \leq \Pi$. Moreover, the condition that $(f \cdot b)(\tau)>0$ for all $\left.\tau \in \Pi\right|_{U}(n-1)$ implies that $\Pi(f)=\Pi$, proving the first implication.

Conversely, suppose that $f$ is concave on $\Delta$ and $\Pi=\Pi(f)$. For each integer $0<$ $k \leq n$ and each $\left.\tau \in \Pi\right|_{U}(k)$ choose an affine function $\ell$ as in (4.29). Choose then a point $x \in \operatorname{relint}(\tau)$ and $\varepsilon>0$ such that, for all $\sigma \in \Pi(f)(k+1)$ with $\sigma \succ \tau$ the condition $x+\varepsilon v_{\sigma \backslash \tau} \in \sigma$ holds. Then for $c \in M_{k+1}^{+}\left(\left.\Pi\right|_{V}\right)$ with $c(\sigma)>0$ for some $\left.\sigma \in \Pi\right|_{V}(k+1)$ with $\sigma \succ \tau$, we have that

$$
(f \cdot c)(\tau)=\frac{1}{\varepsilon} \sum_{\sigma \succ \tau} c(\sigma)\left(f(x)-f\left(x+\varepsilon v_{\sigma \backslash \tau}\right)\right)>\frac{1}{\varepsilon} \sum_{\sigma \succ \tau} c(\sigma)\left(\ell(x)-\ell\left(x+\varepsilon v_{\sigma \backslash \tau}\right)\right)=0,
$$

thanks to the formula in (3.11), the inequality in (4.29), and the fact that $\ell$ is affine. This shows that $f$ is strictly concave on $\Pi$ and completes the proof.

Remark 4.31 The proof of Proposition 4.30 shows that in order to check that a piecewise affine function $f$ defined on a polyhedral complex $\Pi$ on an $n$-dimensional polyhedron $\Delta$ is strictly concave, it suffices to show that $(f \cdot b)(\tau)>0$ for every $\tau \in \Pi(n-1)$. This is not the case when $\Delta$ is replaced by an arbitrary polyhedral space.

Let $\Pi$ be a polyhedral complex on $X$, and $f$ a piecewise affine function on this Euclidean polyhedral space whose restriction to each polyhedron of $\Pi$ is concave. Then we consider the set of polyhedra in $X$ defined as

$$
S(\Pi, f)=\bigcup_{\sigma \in \Pi} \Pi\left(\left.f\right|_{\sigma}\right),
$$

where for each $\sigma \in \Pi$ we denote by $\Pi\left(\left.f\right|_{\sigma}\right)$ the polyhedral complex on $\sigma$ given by the affinity loci of the restriction of $f$ to this polyhedron as in (4.28).

Proposition 4.33 The set $S(\Pi, f)$ is the minimal subdivision of $\Pi$ where the piecewise affine function $f$ is defined.

Proof For each $\tau, \sigma \in \Pi$ with $\tau \prec \sigma$, the restriction to $\tau$ of the polyhedral complex $\Pi\left(\left.f\right|_{\sigma}\right)$ agrees with $\Pi\left(\left.f\right|_{\sigma}\right)$. This implies that $S(\Pi, f)$ is a polyhedral complex on $X$, and Proposition 4.30 implies that it is the minimal subdivision of $\Pi$ where $f$ is defined.

We next show that any polyhedral complex satisfying a finiteness condition admits a regular subdivision.

Theorem 4.34 Let $\Pi$ be a polyhedral complex on $X$ such that $\left.\Pi\right|_{U}$ is finite. Then there is a strongly concave piecewise affine function $f$ on $X$ such that $\left.S(\Pi, f)\right|_{U}$ is finite and $f$ is strictly concave on $\left.S(\Pi, f)\right|_{U}$. In particular, $S(\Pi, f)$ is a subdivision of $\Pi$ that is regular on $U$. 
Proof For each $\left.\sigma \in \Pi\right|_{U}$ choose linear functions $\ell_{\sigma, j}$ on $N_{X}, j=1, \ldots, m_{\sigma}$, defining the polyhedron $\iota_{X}(\sigma)$ as the subset of points $x \in H_{X}$ such that $\ell_{\sigma, j}(x) \geq 0$ for all $j$. Consider the concave piecewise affine function $f_{\sigma}^{\prime}: H_{X} \rightarrow \mathbb{R}$ defined, for $x \in H_{X}$, as

$$
f_{\sigma}^{\prime}(x)=\min \left(0, \ell_{\sigma, 1}(x), \ldots, \ell_{\sigma, m_{\sigma}}(x)\right) .
$$

Set

$$
f^{\prime}=\sum_{\left.\sigma \in \Pi\right|_{U}} f_{\sigma}^{\prime} \quad \text { and } \quad f=f^{\prime} \circ \iota_{X}
$$

Since $\left.\Pi\right|_{U}$ is finite, $f^{\prime}$ is well-defined, concave and piecewise linear. Since $f$ is the pull-back to $X$ of the concave function $f^{\prime}$ on $H_{X}$, it is a strongly concave piecewise affine function on $X$. By Proposition 4.33, we can then consider the associated subdivision $S(\Pi, f)$ of $\Pi$. Вy construction, the restriction of $S(\Pi, f)$ to the open subset $U$ is also finite.

We need to show that $f$ is strictly concave on $S(\Pi, f)$. So let $\left.\tau \in S(\Pi, f)\right|_{U}(k-1)$ with $k \geq 1$. Let $c \in M_{k}^{+}\left(\left.S(\Pi, f)\right|_{U}\right)$ with $c(\sigma)>0$ for some $\left.\sigma \in S(\Pi, f)\right|_{U}(k)$ with $\sigma \succ \tau$. Let $\rho$ be the minimal polyhedron of $\left.\Pi\right|_{U}$ containing $\tau$.

If $\operatorname{dim}(\rho)>\operatorname{dim}(\tau)$ then necessarily $\tau \cap \operatorname{relint}(\rho) \neq \emptyset$ since otherwise $\tau$ would be contained in a face of $\rho$, contradicting the minimality of $\rho$. In this case Proposition 4.30 implies that $(f \cdot c)(\tau)>0$. Else when $\operatorname{dim}(\rho)=\operatorname{dim}(\tau)$, we number as $\sigma_{1}, \ldots, \sigma_{r}$ the polyhedra in $\left.S(\Pi, f)\right|_{V}(k)$ with $\tau \prec \sigma_{i}$ and $c\left(\sigma_{i}\right)>0$. We have that $r>0$ by the hypothesis on $c$. Choose a point $x$ in the relative interior of $\tau$ and $\varepsilon>0$ such that, for $i=1, \ldots, r$,

$$
x_{i}:=x+\varepsilon v_{\sigma_{i} \backslash \tau} \in \operatorname{relint}\left(\sigma_{i}\right) .
$$

Write $\alpha_{i}=c\left(\sigma_{i}\right) / \sum_{j=1}^{r} c\left(\sigma_{j}\right)$. Since $c$ is a Minkowski weight, we have that $\iota_{X}(x)=$ $\sum \alpha_{i} \iota_{X}\left(x_{i}\right)$. Moreover,

$$
(f \cdot c)(\tau)=\frac{\sum_{j} c\left(\sigma_{j}\right)}{\varepsilon}\left(f^{\prime}\left(\iota_{X}(x)\right)-\sum_{i=1} \alpha_{i} f^{\prime}\left(\iota_{X}\left(x_{i}\right)\right)\right) .
$$

Since $\tau \subset \rho, \operatorname{dim}(\tau)=\operatorname{dim}(\rho)$ and $x_{i} \in \operatorname{relint}\left(\sigma_{i}\right)$ where $\operatorname{dim}\left(\sigma_{i}\right)>\operatorname{dim}(\tau)$ and $\sigma_{i} \succ \tau$, we deduce that $x_{i} \notin \rho$. Let $f_{\rho}^{\prime}$ be the function defined as (4.35) for $\rho$. Then $f_{\rho}^{\prime}\left(\iota_{X}(x)\right)=0$ and, for each $i$, we also have that $f_{\rho}^{\prime}\left(\iota_{X}\left(x_{i}\right)\right)<0$ because $x_{i} \notin \rho$. This implies that

$$
f_{\rho}^{\prime}(\iota(x))-\sum_{i=1} \alpha_{i} f_{\rho}^{\prime}\left(\iota\left(x_{i}\right)\right)>0
$$

Since $f^{\prime}$ is obtained from $f_{\rho}^{\prime}$ by adding to it a concave piecewise affine function, we deduce that

$$
f^{\prime}(\iota(x))-\sum_{i=1} \alpha_{i} f^{\prime}\left(\iota\left(x_{i}\right)\right)>0 .
$$

Hence $f$ is strictly concave on $\left.S(\Pi, f)\right|_{U}$ and $S(\Pi, f)$ is a subdivision of $\Pi$ that is regular on $U$, as stated.

Theorem 4.37 Let $\Pi$ be a polyhedral complex on $X$ such that $\left.\Pi\right|_{U}$ is finite. Then there is a subdivision $\Pi^{\prime}$ of $\Pi$ such that for every piecewise affine function $g \in \mathrm{PA}_{\Pi^{\prime}}(U)$ there are concave piecewise affine functions $f_{1}, f_{2} \in \mathrm{CPA}_{\Pi^{\prime}}(U)$ with

$$
g=f_{1}-f_{2} .
$$


Proof By Theorem 4.34, there is a subdivision $\Pi^{\prime}$ of $\Pi$ and a strictly concave function $f$ on $\left.\Pi^{\prime}\right|_{U}$. The set $\mathrm{PA}_{\Pi^{\prime}}(U)$ is a finite dimensional vector space, and $\mathrm{CPA}_{\Pi^{\prime}}(U)$ is a cone inside it. For each $k \in \mathbb{Z}_{\geq 0}$ and $\tau \in \Pi(k-1)$ choose an open subset $V \subset U$ such that $\tau$ is the only polyhedron of $\Pi(k-1)$ intersecting $V$. Each Minkowski weight $c \in M_{k}\left(\left.\Pi\right|_{V}\right)$ gives a functional $\ell_{k, \tau, V, c}: \mathrm{PA}_{\Pi^{\prime}}(U) \rightarrow \mathbb{R}$ defined, for $g \in \mathrm{PA}_{\Pi^{\prime}}(U)$, by

$$
\ell_{k, \tau, V, c}(g)=(g \cdot c)(\tau) \text {. }
$$

Since $f$ is strictly concave on $\left.\Pi\right|_{V}$, we have that $\ell_{k, \tau, V, c}(f)>0$ for all $k, \tau, V$ and $c$ as above. Hence $f$ lies in the interior of the cone $\mathrm{CPA}_{\Pi^{\prime}}(U)$, and so the interior of this cone is nonempty. Thus

$$
\mathrm{PA}_{\Pi^{\prime}}(U)=\mathrm{CPA}_{\Pi^{\prime}}(U)-\mathrm{CPA}_{\Pi^{\prime}}(U)
$$

which gives the result.

Remark 4.39 The class of concave piecewise affine functions satisfies two fundamental properties, as it follows from its definition and Theorem 4.37:

(1) they preserve the positivity of Minkowski weights,

(2) piecewise affine functions can be written as a difference of concave piecewise affine functions.

By contrast, strongly concave piecewise affine functions do not satisfy the condition (2) and weakly concave functions do not satisfy the condition (1), as it can be verified in the situation of Example 4.17

\section{Concave functions on polyhedral spaces}

In this section we given different notions of concavity for functions on polyhedral spaces, extending the previous notions for piecewise affine functions. We denote by $X$ a quasiembedded polyhedral space with quasi-embedding $\iota: X \rightarrow N$, and $U$ an open subset of it.

First we first introduce notions of convex combinations of points.

Definition 5.1 A convex combination in $U$ is a triple

$$
\left(x,\left(x_{i}\right)_{i \in I},\left(v_{i}\right)_{i \in I}\right)
$$

with $x \in U$, and where $\left(x_{i}\right)_{i \in I}$ and $\left(v_{i}\right)_{i \in I}$ respectively denote finite collections of points of $U$ and of nonnegative real numbers satisfying

$$
\sum_{i \in I} v_{i}=1 \quad \text { and } \quad \sum_{i \in I} v_{i} \iota\left(x_{i}\right)=\iota(x) .
$$

A convex combination in $U$ is polyhedral if there is a polyhedral complex $\Pi$ on $X$ and polyhedra $\tau \in \Pi$ with $x \in \tau$ and $\sigma_{i} \in \Pi, i \in I$, such that $\sigma_{i} \succ \tau, \sigma_{i} \subset U$ and $x_{i} \in \sigma_{i}$.

If $X$ is Euclidean and of pure dimension, and $U$ is balanced, a convex combination in $U$ is balanced if it is polyhedral and, with notation as above and setting $k=\operatorname{dim}(\tau)+1$, satisfies the following conditions:

(1) the polyhedra $\sigma_{i}, i \in I$, are the different polyhedra in $\Pi(k)$ having $\tau$ as a face, 

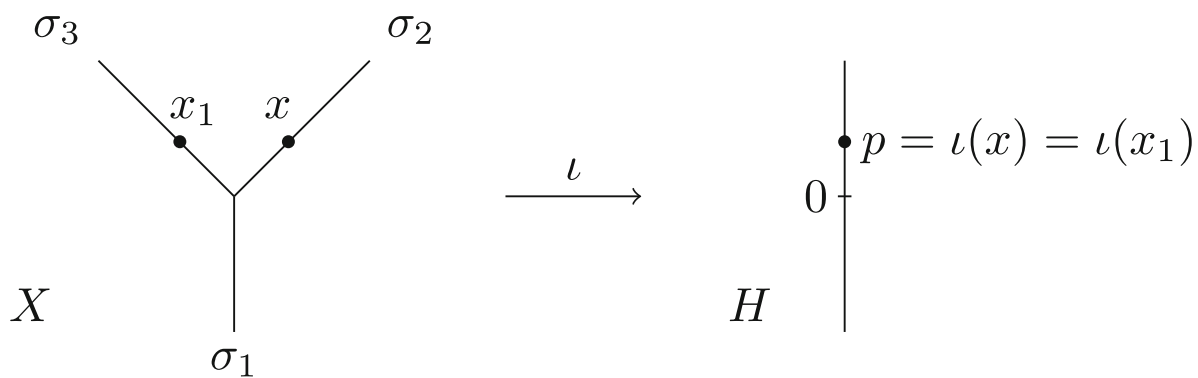

Fig. 3 A convex combination that is not polyhedral
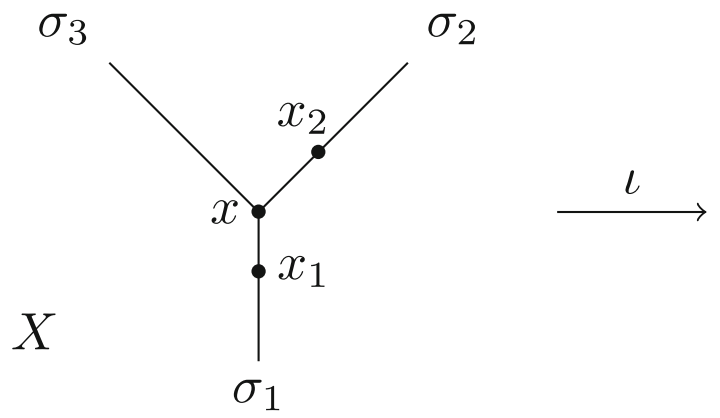

Fig. 4 A convex combination that is polyhedral but not balanced

(2) there is a $\beta_{U}$-positive Minkowski weight $c \in M_{k}\left(\left.\Pi\right|_{U}\right)$ (Definition 4.20) such that $\nu_{i} d\left(x_{i}, \tau\right)=c\left(\sigma_{i}\right), i \in I$, where $d\left(x_{i}, \tau\right)$ denotes the distance between the point $\iota\left(x_{i}\right)$ and the affine subspace $\iota_{\tau}\left(H_{\tau}\right)$ of $N$.

We next give examples illustrating the distinction between the different notions of convex combination.

Example 5.2 We place ourselves in the setting of Example 4.17. For $p \in \mathbb{R}_{>0}$ set

$$
x=(p, 2), \quad x_{1}=(p, 3) \quad \text { and } \quad v_{1}=1 .
$$

Then $\left(x,\left(x_{1}\right),\left(v_{1}\right)\right)$ is a convex combination since $\iota(x)=\iota\left(x_{1}\right)=p$ and so we have that $\sum_{i=1}^{1} v_{i} \iota\left(x_{i}\right)=\iota(x)$. However, this convex combination is not polyhedral: there is no polyhedral complex on $X$ such that $x_{1}$ lies in a polyhedron with a face containing the point $x$.

Set also

$$
x=(0,1), \quad x_{1}=(p, 1), \quad x_{2}=(p, 2) \quad \text { and } \quad v_{i}=\frac{1}{2}, \quad i=1,2 .
$$

This is a polyhedral convex combination for the polyhedral complex $\Pi$ in Example 4.17: we have that $x \in \tau$ and $x_{i} \in \sigma_{i}, i=1,2$, and so $\sum_{i=1}^{2} v_{i} \iota\left(x_{i}\right)=0=\iota(x)$. This polyhedral convex combination is not balanced: setting $\nu_{3}=0$, the vectors

$$
\left(v_{i} d\left(x_{i}, x\right)\right)_{i=1,2,3}=\left(\frac{p}{2}, \frac{p}{2}, 0\right) \quad \text { and } \quad\left(b\left(\sigma_{i}\right)\right)_{i=1,2,3}=(2,1,1)
$$

are not proportional. 

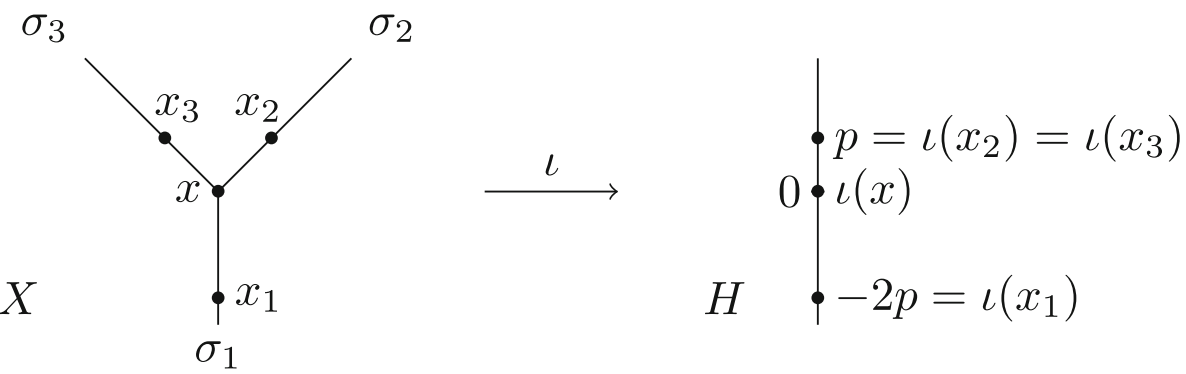

Fig. 5 A balanced convex combination

Finally, the points and positive real numbers

$$
x=(0,1), \quad x_{1}=(2 p, 1), \quad x_{2}=(p, 2), \quad x_{3}=(p, 3) \quad \text { and } \quad v_{i}=\frac{1}{3}, i=1,2,3,
$$

do form a balanced convex combination in $X$.

The basic example of balanced convex combination arises when the $\beta_{U}$-positive Minkowski cycle in Definition 5.1 is the balancing condition itself.

Example 5.3 Suppose that $X$ is Euclidean, of pure dimension $n$ and balanced, and let $\Pi$ be a polyhedral complex on $X$ with balancing condition $b$. For $\tau \in \Pi(n-1)$ let $\sigma_{i}, i=1, \ldots, r$, be the different polyhedra in $\Pi(n)$ having $\tau$ as a facet and set

$$
v_{i}=\frac{b\left(\sigma_{i}\right)}{\sum_{i=1}^{r} b\left(\sigma_{i}\right)}, \quad i=1, \ldots, r .
$$

Let $x \in \operatorname{relint}(\tau)$ and $\left(x,\left(y_{i}\right)_{1 \leq i \leq r},\left(v_{i}\right)_{1 \leq i \leq r}\right)$ a convex combination contained in $\operatorname{relint}(\tau)$. Let $\varepsilon>0$ so that the point

$$
x_{i}:=y_{i}+\varepsilon v_{\sigma_{i} \backslash \tau}
$$

lies in $\sigma_{i}$ for all $i$. Then $\left(x,\left(x_{i}\right)_{1 \leq i \leq r},\left(v_{i}\right)_{1 \leq i \leq r}\right)$ is a balanced convex combination.

Remark 5.4 The construction in Example 5.3 provides balanced convex combinations centered at any point in the relative interior of a polyhedron of dimension $n-1$. Considering arbitrary $\beta_{U}$-positive Minkowski weights as in Definition 5.1 allows to produce balanced convex combinations centered at any point of $U$.

Definition 5.5 Let $f: U \rightarrow \mathbb{R}$ be a function. Then $f$ is strongly concave if for every convex combination in $U$, the inequality

$$
f(x) \geq \sum_{i \in I} v_{i} f\left(x_{i}\right)
$$

holds. It is locally strongly concave if there is an open covering $U=\bigcup_{i} U_{i}$ such that $\left.f\right|_{U_{i}}$ is strongly concave for every $i$.

The function $f$ is concave if the inequality (5.6) is satisfied for all polyhedral convex combinations in $U$.

If $X$ is a Euclidean polyhedral space of pure dimension and $U$ is balanced, then $f$ is weakly concave when this inequality is satisfied for all balanced convex combinations in $U$. 
The next result shows that Definition 5.5 generalizes the notions of concavity for piecewise affine functions from Definitions 4.2 and 4.6.

Proposition 5.7 Let $f$ be a piecewise affine function on $U$. Then $f$ is strongly concave in the sense of Definition 4.2 (respectively concave, weakly concave in the sense of Definition 4.6) if and only if it is strongly concave (respectively concave, weakly concave) in the sense of Definition 5.5 .

Proof We prove separately each implication.

Strong concavity in the sense of Definition 4.2 implies strong concavity in the sense of Definition 5.5:

Let $f$ be strongly concave in the sense of Definition 4.2. Then there is a function

$$
f^{\prime}: N \longrightarrow \mathbb{R} \cup\{ \pm \infty\}
$$

that is concave in the sense of Definition 4.1 and such that $f=\left.f^{\prime} \circ \iota\right|_{U}$. Since $f$ takes only finite values, then $f^{\prime}$ also takes only finite values on $\iota(U)$. Given a convex combination $\left(x,\left(x_{i}\right)_{i \in I},\left(v_{i}\right)_{i \in I}\right)$ in $U$, the concavity of $f^{\prime}$ and this finiteness condition imply that

$$
f(x)=\left(f^{\prime} \circ \iota\right)(x)=f^{\prime}\left(\sum_{i \in I} v_{i} \iota\left(x_{i}\right)\right) \geq \sum_{i \in I} v_{i} f^{\prime}\left(\iota\left(x_{i}\right)\right)=\sum_{i \in I} v_{i} f\left(x_{i}\right),
$$

and so $f$ is strongly concave in the sense of Definition 5.5.

Strong concavity in the sense of Definition 5.5 implies strong concavity in the sense of Definition 4.2:

Let now $f$ be strongly concave in the sense of Definition 5.5. Let $g: \iota(U) \rightarrow \mathbb{R}$ be the function defined, for $y \in \iota(U)$, by

$$
g(y)=f(x)
$$

for any $x \in U$ with $\iota(x)=y$. It is well-defined, because for $x_{1}, x_{2} \in U$ such that $\iota\left(x_{1}\right)=\iota\left(x_{2}\right)$ we have that $\left(x_{1},\left(x_{2}\right),(1)\right)$ is a convex combination in $U$ and so $f\left(x_{1}\right) \geq f\left(x_{2}\right)$. Similarly $f\left(x_{2}\right) \geq f\left(x_{1}\right)$, and so $f\left(x_{1}\right)=f\left(x_{2}\right)$.

Consider the function $f^{\prime}: H \rightarrow \mathbb{R} \cup\{ \pm \infty\}$ defined, for $y \in H$, by

$$
f^{\prime}(y)=\sup \sum_{i \in I} v_{i} g\left(y_{i}\right)
$$

the supremum being over the collections of points $\left(y_{i}\right)_{i \in I}$ in $\iota(U)$ and of nonnegative real numbers $\left(v_{i}\right)_{i \in I}$ with $\sum_{i \in I} v_{i}=1$ and $\sum_{i \in I} v_{i} y_{i}=y$. In particular, if $y$ does not belong to the convex hull of $\iota_{X}(U)$ then $f^{\prime}(y)=-\infty$.

By construction, the hypograph of $f^{\prime}$ coincides with the convex hull of the subset $\{(y, z) \mid$ $y \in \iota(U), z \leq g(y)\}$ of $H \times \mathbb{R}$. In particular, this hypograph is a convex set and so $f^{\prime}$ is concave in the the sense of Definition 4.1.

We claim that $f=\left.f^{\prime} \circ \iota\right|_{U}$. To prove this, let $x \in U$ and set $y=\iota(x)$. On the one hand, let $\left(y_{i}\right)_{i \in I}$ and $\left(v_{i}\right)_{i \in I}$ as in (5.8) and write $y_{i}=\iota\left(x_{i}\right)$ with $x_{i} \in U, i \in I$. Then $\left(x,\left(x_{i}\right)_{i \in I},\left(v_{i}\right)_{i \in I}\right)$ is a convex combination in $U$ and so

$$
f(x) \geq \sum_{i \in I} v_{i} f\left(x_{i}\right)=\sum_{i \in I} v_{i} g\left(y_{i}\right)
$$

which implies that $f^{\prime}(y) \leq f(x)$. On the other hand, choosing the collections $(y)$ and (1) in the definition of $f^{\prime}$ in (5.8), we deduce that

$$
f^{\prime}(y) \geq f(x)
$$


and so $f^{\prime}(y)=f(x)$. Hence $f=\left.f^{\prime} \circ \iota\right|_{U}$ and so $f$ is strongly concave in the sense of Definition 4.2.

Concavity in the sense of Definition 4.6 implies concavity in the sense of Definition 5.5:

Now suppose that $f$ is concave in the sense of Definition 4.6 and consider a polyhedral convex combination $\left(x,\left(x_{i}\right)_{i \in I},\left(v_{i \in I}\right)\right)$ in $U$. By definition, there is a polyhedral complex $\Pi$ on $X$ and polyhedra $\tau \in \Pi$ with $x \in \tau$ and $\sigma_{i} \in \Pi, i \in I$, such that $\sigma_{i} \succ \tau, \sigma_{i} \subset U$ and $x_{i} \in \sigma_{i}$.

Choose a subdivision $\Pi^{\prime}$ of $\Pi$ where $f$ is defined and such that $x$ is a vertex of $\left.\Pi^{\prime}\right|_{U}$ and, for each $i \in I$, there is a segment $\rho_{i}$ in $\left.\Pi^{\prime}\right|_{U}$ having $x$ as a vertex and pointing in the direction of $x_{i}$ from $x$. Choose also $\varepsilon>0$ such that the point

$$
y_{i}=x+\varepsilon\left(x_{i}-x\right)
$$

lies in $\rho_{i}$. Let $V \subset U$ be an open neighborhood of $x$ such that $\left.\Pi^{\prime}\right|_{V}(0)=\{x\}$ and $c$ the positive 1-dimensional weight on $\left.\Pi^{\prime}\right|_{V}$ given, for $\left.\rho \in \Pi^{\prime}\right|_{V}$, by

$$
c(\rho)=\sum_{\rho_{i}=\rho} v_{i}\left\|x_{i}-x\right\|,
$$

the sum being over the indexes $i \in I$ such that $\rho_{i}=\rho$. We have that

$$
\sum_{\rho \succ\{x\}} c(\rho) v_{\rho \backslash\{x\}}=\sum_{i \in I} v_{i}\left\|x_{i}-x\right\| v_{\rho_{i} \backslash\{x\}}=\sum_{i \in I} v_{i}\left(x_{i}-x\right)=0
$$

and so $c \in M_{1}^{+}\left(\left.\Pi^{\prime}\right|_{V}\right)$. By Proposition 4.12, $(f \cdot c)(\{x\}) \geq 0$, and so the formula for the product in (3.11) implies that

$$
f(x) \geq \sum_{i \in I} v_{i} f\left(y_{i}\right)
$$

By Corollary 4.24, for each $i \in I$ the piecewise affine function $f$ is convex on $\sigma_{i}$ in the usual sense and so $f\left(y_{i}\right) \geq(1-\varepsilon) f(x)+\varepsilon f\left(x_{i}\right)$. With (5.9), this implies that $f(x) \geq$ $\sum_{i \in I} v_{i} f\left(x_{i}\right)$, proving that $f$ is concave in the sense of Definition 5.5.

Concavity in the sense of Definition 5.5 implies concavity in the sense of Definition 4.6:

Suppose that $f$ is concave in the sense of Definition 5.5. Let $\Pi$ be a polyhedral complex on $X$ where $f$ is defined, $V \subset U$ an open subset, $k \in \mathbb{Z}_{\geq 0}$ and $c \in M_{k}^{+}\left(\left.\Pi\right|_{V}\right)$.

For each $\left.\tau \in \Pi\right|_{V}(k-1)$ let $\sigma_{i}, i \in I$, be the polyhedra of $\left.\Pi\right|_{V}(k)$ having $\tau$ as a face and set

$$
S=\sum_{i \in I} c\left(\sigma_{i}\right)
$$

If $S=0$ then $c\left(\sigma_{i}\right)=0$ for all $i \in I$ because $c$ is positive, and therefore $(f \cdot c)(\tau)=0$ in this case. Else $S>0$, and then we choose $x \in \operatorname{relint}(\tau)$ and $\varepsilon>0$ so that for each $i \in I$, the point

$$
x_{i}^{\prime}:=\iota(x)+\varepsilon v_{\sigma_{i} \backslash \tau}
$$

lies in $\iota\left(\sigma_{i} \cap V\right)$. Set $x_{i}$ for the unique point in $\sigma_{i} \cap V$ such that $\iota\left(x_{i}\right)=x_{i}^{\prime}$. Then $\left(x,\left(x_{i}\right)_{i \in I},\left(c\left(\sigma_{i}\right) / S\right)_{i \in I}\right)$ is a polyhedral convex combination in $V$ and so $f(x) \geq$ $\sum_{i=1}^{r} \frac{c\left(\sigma_{i}\right)}{S} f\left(x_{i}\right)$. The formula in (3.11) then implies that

$$
(f \cdot c)(\tau)=\frac{S}{\varepsilon}\left(f(x)-\sum_{i \in I} \frac{c\left(\sigma_{i}\right)}{S} f\left(x_{i}\right)\right) \geq 0 .
$$


We deduce that $f \cdot c \in M_{k-1}^{+}\left(\left.\Pi\right|_{V}\right)$ and so Proposition 4.12 implies that $f$ is concave in the sense of Definition 4.6.

Weak concavity in the sense of Definition 4.6 implies weak concavity in the sense of Definition 5.5

Suppose now that $f$ is weakly concave in the sense of Definition 4.6 and let $\left(x,\left(x_{i}\right)_{i \in I},\left(v_{i}\right)_{i \in I}\right)$ be a balanced convex combination as in Definition 5.1. With notation as in that latter definition, let $\Pi^{\prime}$ be a subdivision of $\Pi$ where $f$ is defined and choose a polyhedron $\tau^{\prime} \in \Pi^{\prime}(k-1)$ with $x \in \tau^{\prime} \subset \tau$. For each $i \in I$ there is a unique $\sigma_{i}^{\prime} \in \Pi^{\prime}(k)$ with $\tau^{\prime} \prec \sigma_{i}^{\prime}$ and $\sigma_{i}^{\prime} \subset \sigma_{i}$. Consider the subset $K=\bigcup_{i} \sigma_{i}$ and let $g: K \rightarrow \mathbb{R}$ be the unique function such that the restriction $\left.g\right|_{\sigma_{i}}$ is affine and agrees with $f$ in the smaller polyhedra $\sigma_{i}^{\prime}$, that is

$$
\left.g\right|_{\sigma_{i}^{\prime}}=\left.f\right|_{\sigma_{i}^{\prime}}
$$

for all $i$. Since $x \in \tau^{\prime} \subset \sigma_{i}^{\prime}$ for all $i$, we have that $g(x)=f(x)$. By Corollary 4.24 we have that $f$ is concave on $\sigma_{i}$ in the usual sense, and so $g(y) \geq f(y)$ for all $y \in K$. Moreover

$$
g(x)-\sum_{i \in I} v_{i} g\left(x_{i}\right)=(f \cdot c)\left(\tau^{\prime}\right) \geq 0 .
$$

We deduce that $f(x)=g(x) \geq \sum_{i \in I} v_{i} g\left(x_{i}\right) \geq \sum_{i \in I} v_{i} f\left(x_{i}\right)$, and so $f$ is weakly concave in the sense of Definition 5.5.

Weak concavity in the sense of Definition 5.5 implies weak concavity in the sense of Definition 4.6:

Finally assume that $f$ is weakly concave in the sense of Definition 5.5 . Let $\Pi$ be a polyhedral complex on $X$ where $f$ is defined and denote by $b_{U}$ the balancing condition on $\left.\Pi\right|_{U}$.

For each $\left.\tau \in \Pi\right|_{U}(n-1)$ let $\sigma_{i}, i \in I$, be the maximal polyhedra of $\Pi$ having $\tau$ as a facet and set $S=\sum_{i \in I} b_{U}\left(\sigma_{i}\right)$. Choose $x \in \operatorname{relint}(\tau)$ and $\varepsilon>0$ so that, for each $i \in I$, the point

$$
x_{i}^{\prime}:=\iota(x)+\varepsilon v_{\sigma_{i} \backslash \tau}
$$

lies in $\iota\left(\sigma_{i} \cap U\right)$. Set $x_{i}$ for the only point in $\sigma_{i} \cap U$ such that $\iota\left(x_{i}\right)=x_{i}^{\prime}$. Then $\left(x,\left(x_{i}\right)_{i \in I},\left(b_{U}\left(\sigma_{i}\right) / S\right)_{i \in I}\right)$ is a balanced convex combination, which gives the inequality $f(x) \geq \sum_{i=1}^{r} \frac{b_{U}\left(\sigma_{i}\right)}{S} f\left(x_{i}\right)$. Hence

$$
\left(f \cdot b_{U}\right)(\tau)=\frac{S}{\varepsilon}\left(f(x)-\sum_{i=1}^{r} \frac{b_{U}\left(\sigma_{i}\right)}{S} f\left(x_{i}\right)\right) \geq 0
$$

and so $f$ is weakly concave in the sense of Definition 4.6.

The next result extends Corollary 4.24 to concave functions that are not necessarily piecewise affine.

Proposition 5.10 Let $X$ be a Euclidean polyhedral space of pure dimension, $U$ a balanced open subset of $X$, and $f$ a weakly concave function on $U$. Let $\Pi$ be a polyhedral complex on $X$ and $\sigma \in \Pi$ a polyhedron contained in $U$. Then the restriction $\left.f\right|_{\sigma}$ is concave in the usual sense.

Proof We assume without loss of generality that $\sigma$ is of maximal dimension. Let $x, y \in \sigma$ be different points and $0<t<1$ a real number, and set $z=t x+(1-t) y$. Since $\iota$ is injective on $\sigma$, we have that $\iota(x) \neq \iota(y)$. 
Consider the function $g^{\prime}: H \rightarrow \mathbb{R}$ defined by

$$
g^{\prime}(u)=\min (0,\langle\iota(z)-\iota(y), u-\iota(z)\rangle)
$$

and set $g=g^{\prime} \circ \iota$. This is a strongly concave piecewise affine function that takes the value 0 at $x$ and at $z$, and is strictly negative at $y$. Let $S(\Pi, g)$ be the subdivision of $\Pi$ associated to $g$ as in (4.32) and denote by $b_{U}$ the pullback to this subdivision of the balancing condition on $U$. In this subdivision, $\sigma$ gets broken into two polyhedra of maximal dimension $\sigma_{1}$ and $\sigma_{2}$ such that $x \in \sigma_{1}, y \in \sigma_{2}$ and $z \in \sigma_{1} \cap \sigma_{2}$. Moreover $b_{U}\left(\sigma_{1}\right)=b_{U}\left(\sigma_{2}\right)=b_{U}(\sigma)>0$.

Since $t d(x, z)=t(1-t) d(x, y)=(1-t) d(y, z)$ and the points $x, z, y$ are aligned, the triple $(z,(x, y),(t, 1-t))$ is a balanced convex linear combination in $U$. Since $f$ is weakly concave on $U$,

$$
f(z) \geq t f(x)+(1-t) f(y)
$$

and so $\left.f\right|_{\sigma}$ is concave, as stated.

As in the classical case, the three notions of concavity are stable under sums, product by a positive number and taking infimum. The next proposition follows directly from the definitions.

Proposition 5.11 Let $f_{1}$ and $f_{2}$ be strongly concave (respectively concave, weakly concave) functions on $U$ and $\alpha \in \mathbb{R}_{\geq 0}$. Then $\alpha f_{1}$ and $f_{1}+f_{2}$ are also strongly concave (respectively concave, weakly concave) functions on $U$.

Proposition 5.12 Let $\left\{f_{\lambda}\right\}_{\lambda \in \Lambda}$ be a family of strongly concave (respectively concave, weakly concave) functions on $U$. If $\inf _{\lambda \in \Lambda} f_{\lambda}(x)>-\infty$ for all $x \in U$, then $\inf _{\lambda \in \Lambda} f_{\lambda}$ is also a strongly concave (respectively concave, weakly concave) function on $U$.

Proof Set for short $f=\inf _{\lambda \in \Lambda} f_{\lambda}$. Suppose that each function $f_{\lambda}$ is strongly concave and consider a convex combination $\left(x,\left(x_{i}\right)_{i \in I},\left(v_{i}\right)_{i \in I}\right)$ in $U$. We have that

$$
f_{\lambda}(x) \geq \sum_{i \in I} v_{i} f_{\lambda}\left(x_{i}\right)
$$

and so

$$
f(x)=\inf _{\lambda \in \Lambda} f_{\lambda}(x) \geq \inf _{\lambda \in \Lambda} \sum_{i \in I} v_{i} f_{\lambda}\left(x_{i}\right) \geq \sum_{i \in I} v_{i} \inf _{\lambda \in \Lambda} f_{\lambda}\left(x_{i}\right)=\sum_{i \in I} v_{i} f\left(x_{i}\right),
$$

which shows that $f$ is strongly concave. The proofs for concave and weakly concave functions are done in a similar way.

We next show that the conditions of being concave and weakly concave are local.

Proposition 5.13 Let $f: U \rightarrow \mathbb{R}$ be a function and $U=\bigcup_{\lambda} U_{\lambda}$ an open covering. Then $f$ is concave (respectively weakly concave) if and only if $\left.f\right|_{U_{\lambda}}$ is concave (respectively weakly concave) for all $\lambda$.

Proof We give the proof for concave functions. The case of weakly concave functions can be treated similarly, by adding the condition that the considered polyhedral convex combination are balanced.

If $f$ is concave on $U$ then clearly it is also concave on $U_{\lambda}$ for all $\lambda$, and so we only have to consider the reverse implication. Hence we suppose that $\left.f\right|_{U_{\lambda}}$ is concave for all $\lambda$. Let then

$$
s=\left(x,\left(x_{i}\right)_{i \in I},\left(v_{i}\right)_{i \in I}\right)
$$


be a polyhedral convex combination in $U$, and $\tau$ and $\sigma_{i}, i \in I$, the polyhedra appearing in the definition of such a convex combination. Since the classical notion of concavity is local and each polyhedron $\sigma_{i}$ is contained in $U$, Proposition 5.10 implies that $f$ is concave in the usual sense on $\sigma_{i}$. As the segment $\overline{x x_{i}}$ is contained in $\sigma_{i}$, this implies that $f$ is concave in the usual sense on $\overline{x x_{i}}$.

For $\varepsilon \in[0,1]$ the point $x+\varepsilon\left(x_{i}-x\right)$ lies in $U$ for all $i \in I$, and so the triple

$$
\varepsilon s=\left(x,\left(x+\varepsilon\left(x_{i}-x\right)\right)_{i \in I},\left(v_{i}\right)_{i \in I}\right)
$$

is also a polyhedral convex combination in $U$.

Let $\lambda$ such that $x \in U_{\lambda}$. Since $U_{\lambda}$ is open, there is $0<\varepsilon \leq 1$ such that $\varepsilon s$ is a polyhedral convex combination in $U_{\lambda}$. Since $f$ is concave on $U_{\lambda}$ in the sense of Definition 5.5 and $f$ is concave in the usual sense on $\overline{x x_{i}}$ for each $i \in I$, then

$$
f(x) \geq \sum_{i} v_{i} f\left(x+\varepsilon\left(x_{i}-x\right)\right) \geq \sum_{i} v_{i}(1-\varepsilon) f(x)+\sum_{i} v_{i} \varepsilon f\left(x_{i}\right) .
$$

This readily implies that $f(x) \geq \sum_{i} v_{i} f\left(x_{i}\right)$ and so $f$ is concave.

As shown in Example 4.3, the property of being strongly concave is not local. The argument in the proof of Proposition 5.13 relied on the fact that if $s$ is a polyhedral convex combination, so is the triple $\varepsilon s$ defined in (5.14). This property fails for arbitrary convex combinations, as in the case of that example.

Remark 5.15 An alternative approach to define concavity on polyhedral spaces would be in terms of double polars of cones of concave piecewise affine functions. More precisely, we say that a signed Radon measure $\mu$ on $U$ with compact support is convex if for every concave piecewise affine function $f$ on $U$ the inequality

$$
\int f d \mu \geq 0
$$

is satisfied. If $U$ is balanced, this measure $\mu$ is strongly convex if the inequality (5.16) is satisfied for every weakly concave piecewise affine function $f$ on $U$. A function $f: U \rightarrow \mathbb{R}$ is $*$-concave if the inequality (5.16) is satisfied for every convex measure $\mu$, and it is weakly $*$-concave if the inequality is satisfied for every strongly convex measure $\mu$.

By Proposition 5.7, any polyhedral (respectively, balanced) convex combination gives rise to a discrete convex (respectively, strongly convex) measure, and so it is clear that being *-concave (respectively, being weakly $*$-concave) implies being concave (respectively, being weakly concave).

By the bipolar theorem, any $*$-concave function can be approximated by concave piecewise affine functions. By contrast, it is not clear yet whether the notion of being $*$-concave is local, or if the infimum of two $*$-concave functions is also $*$-concave. If one can prove that any concave function can be approximated by concave piecewise affine functions, then every concave function would be $*$-concave and both definitions would agree. Similar considerations can be done concerning weakly concave and weakly $*$-concave. This is still an open question which we hope to continue studying.

\section{Continuity properties}

In this section we study the continuity properties of weakly concave functions on balanced open subsets of polyhedral spaces. We denote by $X$ a Euclidean polyhedral space of pure 
dimension $n$ and $U \subset X$ an open subset that, unless otherwise stated, will be assumed to be balanced, with balancing condition $\beta_{U}$.

Before discussing the different continuity properties we prove a technical lemma.

Lemma 6.1 Let $y \in U$ and let $\Pi$ be a polyhedral complex in $X$ such that the set

$$
V=\bigcup_{\substack{y \in \sigma \in \Pi \\ \sigma \subset U}} \sigma
$$

is a neighborhood of $y$. Then, for each point $x_{0} \in V$ there is a balanced convex combination

$$
\left(y,\left(x_{i}\right)_{0 \leq i \leq r},\left(v_{i}\right)_{0 \leq i \leq r}\right)
$$

centered at $y$, with $x_{0}$ as one of the points of the combination and $\nu_{0}>0$.

Proof Let $\sigma$ be the minimal polyhedron of $\Pi$ containing both $x_{0}$ and $y$. Consider the segment $L=\overline{y x_{0}}$ and choose linear functions $\ell_{j}, j=1, \ldots, m$, on $N_{X}$ defining the image $\iota_{X}(L)$ as the subset of points $x \in H_{X}$ such that $\ell_{j}(x) \geq 0$ for all $j$. Consider the concave piecewise affine function $g^{\prime}: H_{X} \rightarrow \mathbb{R}$ defined, for $x \in H_{X}$, by

$$
g^{\prime}(x)=\min \left(0, \ell_{1}(x), \ldots, \ell_{m}(x)\right)
$$

and set $g=g^{\prime} \circ \iota_{X}$. This is a strongly concave piecewise affine function on $X$ and it determines a subdivision $\Pi^{\prime}:=S(\Pi, g)$ of $\Pi$, as in (4.32).

By construction $\{y\} \in \Pi^{\prime}(0)$ and there is a 1-dimensional polyhedron $\tau_{0} \in \Pi^{\prime}(1)$ having $y$ and $x_{0}$ as vertices. Let $\tau_{i}, i=1, \ldots, r$, be the other 1-dimensional polyhedra of $\Pi^{\prime}(1)$ having $y$ as a vertex. Let $b_{U}$ be the Minkowski weight on $\Pi^{\prime}$ defining the balancing condition $\beta_{U}$ on on $U$ and consider the 1-dimensional $\beta_{U}$-positive Minkowski weight

$$
c=g^{n-1} \cdot b_{U} .
$$

By Proposition 4.30 applied to the polyhedron $\sigma$ and the concave piecewise affine function $\left.g\right|_{\sigma}$, we deduce that $c\left(\tau_{0}\right)>0$.

For $i=1, \ldots, r$ set $x_{i}$ for the vertex of $\tau_{i}$ different from $y$, and put

$$
v_{i}=x_{i}-y \quad \text { and } \quad v_{i}=\frac{c\left(\tau_{i}\right)}{\left\|v_{i}\right\|}, \quad i=0, \ldots, r .
$$

Then the triple $\left(y,\left(x_{i}\right)_{0 \leq i \leq r},\left(v_{i}\right)_{0 \leq i \leq r}\right)$ is a balanced convex combination on $U$ satisfying the conditions of the lemma.

The next result is the analogue of the fact that a concave function on an open subset of $\mathbb{R}^{n}$ is continuous [12, Theorem 10.1].

Theorem 6.2 Let $f$ be a weakly concave function on $U$. Then $f$ is continuous.

Proof Let $y \in X$ and choose a polyhedral complex $\Pi$ on $X$ such that the union of the polyhedra $\sigma \in \Pi$ with $y \in \sigma$ and $\sigma \subset U$ is a neighborhood of $y$. To prove that $f$ is continuous at $y$, it is enough to prove that for each such $\sigma$, the restriction $\left.f\right|_{\sigma}$ is continuous at $y$.

By Proposition 5.10, $\left.f\right|_{\sigma}$ is concave. Let $f^{\prime}=\operatorname{cl}\left(\left.f\right|_{\sigma}\right)$ be its closure, that is, the upper semicontinuous hull of the concave function $\left.f\right|_{\sigma}$, see [12, §7] for the analogous notion for convex functions. The function $f^{\prime}$ is concave and, by [12, Theorem 10.2], it is continuous 
and agrees with $\left.f\right|_{\sigma}$ in the relative interior of $\sigma$. Hence, to prove the continuity of $\left.f\right|_{\sigma}$ at $y$ it is enough to show that $\left.f\right|_{\sigma}(y)=f^{\prime}(y)$.

For $x_{0} \in \operatorname{relint}(\sigma)$ let $\Pi^{\prime}$ be the subdivision of $\Pi$ and $\left(y,\left(x_{i}\right)_{0 \leq i \leq r},\left(v_{i}\right)_{0 \leq i \leq r}\right)$ the balanced convex combination given by Lemma 6.1. For each $i$ write $v_{i}=x_{i}-y$ for the vector spanning the segment $\tau_{i}$. The weak concavity of $f$ then implies that for every $0<\eta \leq 1$,

$$
f(y) \geq \sum_{i=0}^{r} v_{i} f\left(y+\eta v_{i}\right) .
$$

By Proposition 5.10, the restriction of $f$ to each $\tau_{i}$ is concave and so, by [12, Theorem 10.2], it is lower semicontinuous. Hence given $\varepsilon>0$ there is $\eta_{0}>0$ such that for each $0<\eta<\eta_{0}$ and each $0 \leq i \leq r$ we have that

$$
f\left(y+\eta v_{i}\right) \geq f(y)-\varepsilon .
$$

Combining the inequalities (6.3) and (6.4) and the fact that $\sum_{i=0}^{r} v_{i}=1$, we deduce that, for $0<\eta \leq \eta_{0}$,

$$
\begin{aligned}
& f\left(y+\eta v_{0}\right) \leq \frac{1}{v_{0}} f(y)-\sum_{i=1}^{r} \frac{v_{i}}{v_{0}} f\left(y+\eta v_{i}\right) \\
& \leq \frac{1}{v_{0}} f(y)-\sum_{i=1}^{r} \frac{v_{i}}{v_{0}}(f(y)-\varepsilon)=f(y)+\left(\sum_{i=1}^{r} \frac{v_{i}}{v_{0}}\right) \varepsilon .
\end{aligned}
$$

Therefore $\left.f\right|_{\tau_{0}}$ is upper semicontinuous at $y$ and so it is continuous at this point, completing the proof.

Corollary 6.5 Suppose that $X$ is a locally balanceable polyhedral space, $U \subset X$ an open subset that is not necessarily balanced, and $f$ a concave function on $U$. Then $f$ is continuous.

Proof Let $U=\bigcup_{i} U_{i}$ be an open covering of $U$ by balanceable open subsets. Since the condition of being continuous is local, it is enough to show that $\left.f\right|_{U_{i}}$ is continuous for each $i$. Since $f$ is concave, the restriction $\left.f\right|_{U_{i}}$ is weakly concave with respect to any balancing condition on $U_{i}$ and, by Theorem 6.2, it is continuous.

Example 6.6 The condition of being locally balanceable in Corollary 6.5 is necessary for the validity of its conclusion. For instance, the ray $\mathbb{R}_{\geq 0}$ is not locally balanceable (Example 3.28) and the function $f: \mathbb{R}_{\geq 0} \rightarrow \mathbb{R}$ defined by $f(x)=1$ for $x>0$ and $f(0)=0$ is concave but not continuous.

Remark 6.7 The rest of the results of this section are only stated for weakly concave functions on a balanced open subset but as in Corollary 6.5, they all admit a variant for concave functions on an open subset of a locally balanceable polyhedral space.

A consequence of Theorem 6.2 is that weakly concave functions satisfy a minimum principle. The next result extends [12, Theorem 32.1] to balanced open subsets of polyhedral spaces.

Proposition 6.8 Suppose that $U$ is connected and let $f$ be a weakly concave function on $U$. If there is a point $y \in U$ such that $f(y)=\inf _{x \in U} f(x)$, then $f$ is constant. 
Proof Assume that there is a point $y \in U$ realizing the infimum of $f$ on $U$ and set $m=f(y)$. By Theorem 6.2, $f$ is continuous and so $f^{-1}(m)$ is a nonempty closed subset of $U$. Since $U$ is connected, to complete the proof it suffices to show that this subset is also is open.

Choose a polyhedral complex $\Pi$ on $X$ such that every $\sigma \in \Pi$ containing the point $y$, is contained in $U$. Hence the subset $V:=\bigcup_{\sigma \ni y} \sigma$ is a neighborhood of $y$ contained in $U$. By Lemma 6.1 , for each point $x_{0} \in V$ there is a balanced convex combination $\left(y,\left(x_{i}\right)_{0 \leq i \leq r},\left(v_{i}\right)_{0 \leq i \leq r}\right)$ with $v_{0}>0$. Since $f$ is weakly concave, we have that

$$
f(y) \geq \sum_{i=0}^{r} v_{i} f\left(x_{i}\right) .
$$

Moreover, since $\sum_{i=0}^{r} v_{i}=1$ and $f\left(x_{i}\right) \geq f(y), i=0, \ldots, r$, we deduce that $f\left(x_{i}\right)=f(y)$ for all $i$ and, in particular, that $f\left(x_{0}\right)=m$. Hence $f$ is constant in $V$ and so $f^{-1}(m)$ is an open subset, concluding the proof.

Our next objective is to show that a weakly concave function is not just continuous, but Lipschitz continuous on any compact subset of its domain, and that its Lipschitz constant can be bounded in terms of the supremum of the function on a slightly larger open subset. The main technical tool is a variant of [3, Proposition 2.2] and [4, Proposition A.1] stated in Lemma 6.11.

Let $(Z, d)$ be a metric space and $f: V \rightarrow \mathbb{R}$ a function on a subset of it. The Lipschitz constant of $f$ on $V$ with respect to $d$ is defined as

$$
\operatorname{Lip}_{V, d}(f)=\sup _{\substack{x, y \in V \\ x \neq y}} \frac{|f(x)-f(y)|}{d(x, y)} \in \mathbb{R}_{\geq 0} \cup\{+\infty\},
$$

with the convention that, if $V$ consists of a single point, then $\operatorname{Lip}_{V}(f)=0$. A function $f$ is called Lipschitz continuous on $V$ with respect to $d$ if $\operatorname{Lip}_{V, d}(f)<+\infty$.

For a polytope $\tau$ in $X$, the Euclidean structure of $X$ induces a metric on $\tau$. Given a function $f: \tau \rightarrow \mathbb{R}$, we denote by

$$
\operatorname{Lip}_{\tau}(f)
$$

the Lipschitz constant of $f$ on $\tau$ with respect to this metric. Following [3, §2] and [4, Appendix A], given two points $u, v \in \tau$, we also denote by

$$
D_{v} f(u):=\left.\frac{d}{d t}\right|_{t=0^{+}} f((1-t) v+t u)
$$

the derivative of $f$ at $v$ in the direction of $u$, whenever the limit exists. This holds when $f$ is concave, in which case this derivative is as an element of $\mathbb{R} \cup\{+\infty\}$.

Lemma 6.9 There exists a constant $C>0$ depending only on $\tau$ such that, for each continuous concave function $f: \tau \rightarrow \mathbb{R}$ and each subset $A \subset \tau$ such that $\tau \backslash A$ has Lebesgue measure zero, the following estimate holds

$$
C^{-1} \operatorname{Lip}_{\tau}(f) \leq \sup _{\substack{v \in A \\ e \in \tau(0)}}\left|D_{v} f(e)\right| \leq C \operatorname{Lip}_{\tau}(f) .
$$

Proof If $f$ is Lipschitz continuous, this is [4, Lemma A.2]. Otherwise, the argument in the proof of this result also shows that $\sup _{v, e}\left|D_{v} f(e)\right|=+\infty$, and so the inequalities in (6.10) are also satisfied in this case. 
Fig. 6 The derivative of $f$ at $v$ in the direction of $u$

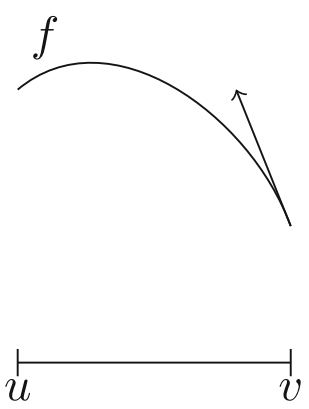

For a function $f: \tau \rightarrow \mathbb{R}$ we set

$$
\|f\|_{C^{0}(\tau)}=\sup _{x \in \tau}|f(x)| \quad \text { and } \quad\|f\|_{C^{0,1}(\tau)}=\|f\|_{C^{0}(\tau)}+\operatorname{Lip}_{\tau}(f) .
$$

Lemma 6.11 There is a constant $C>0$ depending only on $\tau$ such that, for each continuous concave function $f: \tau \rightarrow \mathbb{R}$,

$$
C^{-1}\|f\|_{C^{0,1}(\tau)} \leq\|f\|_{C^{0}(\partial \tau)}+\sup \left|D_{v} f(e)\right| \leq C\|f\|_{C^{0,1}(\tau)},
$$

the supremum being over the facets $F$ of $\tau$, the points $v \in \operatorname{relint}(F)$ and the vertices e of $\tau$ that are not contained in $F$.

Proof If $f$ is Lipschitz continuous, this is [4, Proposition A.1]. Otherwise, Lemma 6.9 implies that $\|f\|_{C^{0,1}(\tau)}=\sup _{F, v, e}\left|D_{v} f(e)\right|=+\infty$, and so the inequalities in (6.12) are also satisfied in this case.

Our next task is to estimate the derivatives $D_{v} f(e)$ that appear in Lemma 6.11 for a polytope in a balanced open subset.

Lemma 6.13 Let $\Pi$ be a polyhedral complex on $X$ such that $\left.\Pi\right|_{U}$ is finite and $\Pi$ is regular on $U$. Then for every polytope $\tau \in \Pi$ contained in $U$ there is a constant $C>0$ such that for every weakly concave function $f: U \rightarrow \mathbb{R}$,

$$
\operatorname{Lip}_{\tau}(f) \leq C\|f\|_{C^{0}(U)}
$$

Proof By possibly shrinking the open subset $U$, we can assume without loss of generality that its closure is compact.

We prove the result by induction on the dimension of $\tau$. If $\operatorname{dim}(\tau)=0$ the result is clear. So we assume that $\operatorname{dim}(\tau)=k \geq 1$ and that the result is true for every polytope of smaller dimension. Let $F$ be a facet of $\tau, v \in \operatorname{relint}(F)$ and $e$ a vertex of $\tau$ not contained in $F$. By Proposition 5.10, $f$ is concave on $\tau$ in the usual sense. This implies that $D_{v}(e) \geq f(e)-f(v)$ and so

$$
-D_{v}(e) \leq 2\|f\|_{C^{0}(\tau)} \leq 2\|f\|_{C^{0}(U)} .
$$

Let $\tau$ and $\sigma_{i}, i=1, \ldots, r$, be the $k$-dimensional polyhedra of $\Pi$ containing $F$. Write $v_{0}=v_{\tau \backslash F}$ and $v_{i}=v_{\sigma_{i} \backslash F}, i=1, \ldots, r$. Put $e_{0}=e$ and for each $i$, pick a vertex $e_{i}$ of $\sigma_{i}$ that is not contained in $F$. Then for $i=0, \ldots, r$ write

$$
e_{i}=v+v_{F, i}+\alpha_{i} v_{i}
$$


with $\alpha_{i}>0$ and $v_{F, i} \in N_{F}$, the vector space associated to $F$ (Definition 2.1).

Let $g$ be a strictly concave function on $\left.\Pi\right|_{U}$ (Definition 4.25), and consider the $\beta_{U}$-positive $k$-dimensional Minkowski weight $c=g^{n-k} \cdot b_{U}$. Set $c_{0}=c(\tau)$ and $c_{i}=c\left(\sigma_{i}\right), i=1, \ldots, r$. Since $c$ is a Minkowski weight,

$$
\sum_{i=0}^{r} \frac{c_{i}}{\alpha_{i}}\left(e_{i}-v\right)=\sum_{i=0}^{r} \frac{c_{i}}{\alpha_{i}} v_{F, i}+\sum_{i=0}^{r} c_{i} v_{i}=\sum_{i=0}^{r} \frac{c_{i}}{\alpha_{i}} v_{F, i} \in N_{F} .
$$

Denote this last vector by $-v_{F}$, which implies pi $=31415$ that $v_{F}+\sum_{i=0}^{r} \frac{c_{i}}{\alpha_{i}} v_{F, i}=0$. Choose $\lambda>0$ small enough so that

$$
p_{F}:=v+\lambda v_{F} \in F \quad \text { and } \quad p_{i}:=v+\lambda \frac{c_{i}}{\alpha_{i}}\left(e_{i}-v\right) \in V, \quad i=0, \ldots, r .
$$

Then the convex combination in $V$

$$
\left(v,\left(p_{F}, p_{0}, \ldots, p_{k}\right),\left(\frac{1}{r+2}, \ldots, \frac{1}{r+2}\right)\right)
$$

is balanced. Since $f$ is weakly concave, we deduce that

$$
0 \geq D_{v} f\left(p_{F}\right)+\sum_{i=0}^{r} D_{v} f\left(p_{i}\right)=\lambda D_{v} f\left(v_{F}\right)+\sum_{i=0}^{r} \lambda \frac{c_{i}}{\alpha_{i}} D_{v} f\left(e_{i}\right) .
$$

Using the inductive hypothesis on $F$, Lemma 6.9 and the analogues of the inequalities (6.14) for $\sigma_{i} \cap \bar{V}, i=1, \ldots, r$, we deduce that

$$
\begin{aligned}
D_{v} f\left(e_{0}\right) & \leq \sum_{i=1}^{r} \frac{-\alpha_{0} c_{i}}{c_{0} \alpha_{i}} D_{v} f\left(e_{i}\right)-\frac{\alpha_{0}}{c_{0}} D_{v} f\left(v_{F}\right) \\
& \leq \sum_{i=1}^{r} \frac{2 \alpha_{0} c_{i}}{c_{0} \alpha_{i}}\|f\|_{C^{0}(V)}+C_{F} \frac{\alpha_{0}}{c_{0}}\left\|v_{F}\right\|\|f\|_{C^{0}(V)} .
\end{aligned}
$$

In the last inequalities, the $\alpha_{i}$ 's depend on $v$, but they can be extended to a continuous nonvanishing function on the compact set $F$. Therefore they attain a strictly positive minimum and a finite maximum. Similarly, $\left\|v_{F}\right\|$ depends on $v$, but again can be extended to a continuous function on $F$, where it attains a maximum. Joining this discussion with the inequality (6.14), there is a constant $C_{\tau}$ such that

$$
\left.\mid D_{v}(e)\right)\left|\leq C_{\tau}\right| f \|_{C^{0}(V)},
$$

and so the result follows from Lemma 6.11.

We next discuss metric structures on polyhedral spaces.

Definition 6.15 A polyhedral metric on $X$ is a metric induced by an injective morphism of polyhedral spaces of $X$ into a Euclidean vector space. For a compact subset $K \subset X$, a polyhedral metric on $K$ is the restriction to this subset of a polyhedral metric on a polyhedral subspace of $X$ containing it.

Every compact subset of a polyhedral space admits a polyhedral metric and all of these metrics are equivalent, as the next result shows. 
Lemma 6.16 Let $K \subset X$ be a compact subset. Then $K$ admits a polyhedral metric, and for any pair $d, d^{\prime}$ of these metrics on $K$, there is a constant $C>0$ such that, for all $x, y \in K$,

$$
C^{-1} d(x, y) \leq d^{\prime}(x, y) \leq C d(x, y) .
$$

Proof Let $\Pi$ be a simplicial polyhedral complex on $X$, that is, a polyhedral complex such that its polyhedra are simplices. Then $\Pi_{0}=\{\sigma \in \Pi \mid \sigma \cap K \neq \emptyset\}$ is a finite collection of simplices of $\Pi$, and

$$
X_{0}=\bigcup_{\sigma \in \Pi_{0}} \sigma
$$

is a polyhedral subspace of $X$ containing $K$. Let $\mathcal{V}$ be the set of vertices of $\Pi_{0}$ and consider the embedding

$$
X_{0} \hookrightarrow \mathbb{R}^{\mathcal{V}}
$$

that sends each vertex $v \in \mathcal{V}$ to the corresponding vector $e_{v}$ in the standard basis of $\mathbb{R}^{\mathcal{V}}$ and that is affine on the simplices of $X_{0}$. Then the Euclidean metric of $\mathbb{R}^{\mathcal{V}}$ induces a polyhedral metric on $X_{0}$ and, a fortiori on $K$.

By Theorem [10, Theorem 2.18] any polyhedral map between compact polyhedral spaces with polyhedral metrics is Lipschitz. This implies the existence of the constant $C$ satisfying the inequalities (6.17).

Theorem 6.18 Let $K \subset U$ be a compact subset and d a polyhedral metric on it. Then there is a constant $C>0$ such that, for every weakly concave function $f$ on $U$,

$$
\operatorname{Lip}_{K, d}(f) \leq C\|f\|_{C^{0}(U)} .
$$

Proof Without loss of generality we can assume that the closure of $U$ is compact and, by Lemma 6.16, that the polyhedral metric is defined on the closure of $U$. Let $X_{0}$ be a polyhedral subspace of $X$ containing $U$ and $X_{0} \hookrightarrow N^{\prime}$ an injective morphism of polyhedral spaces into a Euclidean vector space inducing the polyhedral metric $d$ on $\bar{U}$.

Let $\Pi_{0}$ be a polyhedral complex on $X_{0}$, such that every polyhedron $\sigma \in \Pi_{0}$ with $\sigma \cap K \neq \varnothing$ is contained in $U$. By Theorem 4.34 we can assume that $\Pi_{0}$ is regular on $U$. Set

$$
B=\min \left\{d\left(\sigma, \sigma^{\prime}\right) \mid \sigma, \sigma^{\prime} \in \Pi_{0} \text { with } \sigma \cap K, \sigma^{\prime} \cap K \neq \emptyset \text { and } \sigma \cap \sigma^{\prime}=\emptyset\right\}>0 .
$$

Let $x, y \in K$. If there is $\sigma \in \Pi_{0}$ such that $x, y \in \sigma$ then

$$
\frac{|f(x)-f(y)|}{d(x, y)} \leq \operatorname{Lip}_{\sigma, d}(f) \leq C_{0} \operatorname{Lip}_{\sigma}(f),
$$

where $\operatorname{Lip}_{\sigma}(f)$ denotes the Lipschitz constant of $f$ with respect to the metric on $\sigma$ induced by the Euclidean structure of $X$, and $C_{0}>0$ depends only on $X$ and $d$.

If there are $\sigma, \sigma^{\prime} \in \Pi_{0}$ such that $x \in \sigma, y \in \sigma^{\prime}$ and $\sigma \cap \sigma^{\prime}=\emptyset$ then

$$
\frac{|f(x)-f(y)|}{d(x, y)} \leq \frac{2}{B}\|f\|_{C^{0}(K)} .
$$

Finally, if there are $\sigma, \sigma^{\prime} \in \Pi_{0}$ such that $x \in \sigma, y \in \sigma^{\prime}$ and $\sigma \cap \sigma^{\prime} \neq \emptyset$, there is a point $z \in \sigma \cap \sigma^{\prime}$ with $\max \{d(x, z), d(y, z)\} \leq C_{1} d(x, y)$ for a constant $C_{1}>0$ depending only 
on $X$ and $d$. Then

$$
\begin{aligned}
\frac{|f(x)-f(y)|}{d(x, y)} & \leq \frac{|f(x)-f(z)|}{d(x, y)}+\frac{|f(z)-f(y)|}{d(x, y)} \\
& \leq C_{1} \frac{|f(x)-f(z)|}{d(x, z)}+C_{1} \frac{|f(z)-f(y)|}{d(z, y)} \\
& \leq 2 C_{1} C_{0}\left(\operatorname{Lip}_{\sigma}(f)+\operatorname{Lip}_{\sigma^{\prime}}(f)\right) .
\end{aligned}
$$

It follows from (6.19), (6.20) and (6.21) that

$$
\operatorname{Lip}_{K, d}(f) \leq C\left(\sup _{\substack{\sigma \in \prod_{0} \\ \sigma \cap K \neq \emptyset}} \operatorname{Lip}_{\sigma}(f)+\|f\|_{C^{0}(K)}\right)
$$

for a constant $C>0$ depending only on $X$ and $d$. The result then follows from Lemma 6.13.

As a consequence we obtain the following stronger version of Theorem 6.2, extending [12, Theorem 10.4].

Corollary 6.22 Let $f$ be a weakly concave function on $U$. Then $f$ is Lipschitz continuous on any compact subset of $U$.

The following is the analogue of [12, Theorem 10.6] for weakly concave functions on a balanced open subset of a polyhedral space.

Theorem 6.23 Let $\left\{f_{i}\right\}_{i \in I}$ be a family of weakly concave functions on $U$ and $C^{\prime}, C^{\prime \prime} \subset U$ dense subsets such that $\inf _{i \in I} f_{i}(x)$ and $\sup _{i \in I} f_{i}(x)$ are finite for every $x \in C^{\prime}$ and $x \in C^{\prime \prime}$, respectively. Then $\left\{f_{i}\right\}_{i \in I}$ is uniformly bounded and equi-Lipschitz on any compact subset of $U$.

Proof Let $K \subset U$ be a compact subset and $V \subset U$ an open subset with compact closure containing $K$. By Theorem 6.18, if the family $\left\{f_{i}\right\}_{i \in I}$ is uniformly bounded on $V$ then it is equi-Lipschitz in $K$. So we only need to prove that this family is uniformly bounded on $V$.

For $x \in U$ set $f(x)=\inf _{i} f_{i}(x) \in \mathbb{R} \cup\{-\infty\}$. Let $\Pi$ be a polyhedral complex on $X$. For every $\tau \in \Pi(n)$, the functions $\left.f_{i}\right|_{\tau}$ are concave in the classical sense and since $C^{\prime}$ is dense in $\tau$, [12, Theorem 10.6] implies that $f(x)>-\infty$ for $x \in \operatorname{relint}(\tau)$.

For $x \in X$, there is a balanced convex combination $\left(x,\left(x_{i}\right),\left(v_{i}\right)\right)$ such that the points $x_{i}$ belong to the relative interior of a polyhedron in $\Pi(n)$. Therefore

$$
f(x) \geq \sum_{i} v_{i} f\left(x_{i}\right)>-\infty .
$$

By Proposition 5.12, $f$ is a weakly concave function on $U$ and by Theorem 6.2 it is continuous on $U$. Hence $f$ is uniformly bounded in $K$, which implies that the family $\{f i\}_{i \in I}$ is uniformly bounded below in $K$.

Making $K$ bigger and $\Pi$ finer, we can assume that $K$ is a finite union of polyhedra of $\Pi$ of maximal dimension contained in $V$. For each $\tau \in \Pi(n)$ with $\tau \subset K$ there is a point $x \in C^{\prime \prime} \cap \operatorname{relint}(\tau)$. We then follow the proof of [12, Theorem 10.6]. Choose $\varepsilon$ such that $x+\varepsilon \mathbb{B} \subset \tau$, where $\mathbb{B}$ denotes the unit ball. Since $x \in C^{\prime \prime}$, there is $\alpha \in \mathbb{R}$ such that $f_{i}(x) \leq \alpha$ for all $i$. Moreover, since we have already proved the existence of a uniform lower bound, there is also $\beta \in \mathbb{R}$ such that $f(z) \geq \beta$ for all $z \in \tau$. 
Let now $y \in \tau$ with $y \neq x$. Set

$$
z=x+\frac{\varepsilon}{\|x-y\|}(x-y) \quad \text { and } \quad \lambda=\frac{\varepsilon}{\varepsilon+\|x-y\|} .
$$

Then $z \in x+\varepsilon \mathbb{B} \subset \tau$ and $x=(1-\lambda) z+\lambda y$. Hence, by concavity,

$$
\alpha \geq f_{i}(x) \geq(1-\lambda) f_{i}(z)+\lambda f_{i}(y) \geq(1-\lambda) \beta+\lambda f_{i}(y) .
$$

Thus

$$
f_{i}(y) \leq \frac{\alpha-(1-\lambda) \beta}{\lambda}=\alpha+\frac{\|x-y\|(\alpha-\beta)}{\beta} .
$$

Note that the term on the right hand side is a continuous function with respect to $y$ on the compact set $\tau$. Therefore there is a constant $\gamma_{\tau}$ such that for all $i \in I$ and $y \in \tau$ we have that $f_{i}(y) \leq \gamma_{\tau}$. Since $K$ is compact, the number of maximal polyhedra contained in $K$ is finite. Hence, we obtain a uniform upper bound on the whole of $K$ which concludes the proof.

Theorem 6.23 has the following important consequence, which is an analogue of [12, Theorem 10.8] on polyhedral spaces.

Theorem 6.24 Let $\left(f_{i}\right)_{i \geq 0}$ be a sequence of weakly concave functions on $U$ and $C \subset U a$ dense subset such that, for all $x \in C$, the sequence $f_{i}(x)$ converges to a number $f(x) \in \mathbb{R}$. Then the sequence $\left(f_{i}\right)_{i \geq 0}$ converges to a function $f$ on the whole $U$, the limit function $f$ is weakly concave, and the convergence is uniform on compact subsets.

Proof By Theorem 6.23, the family $\left(f_{i}\right)_{i \geq 0}$ is equi-Lipschitz on each compact subset of $U$. Therefore the statement can be proven as in [12, Theorem 10.8].

On the same spirit, other results of [12, Chapter 10] can be translated to the current setting. For instance we particularize the analogue of [12, Theorem 10.7]. It is a consequence of theorems 6.18 and 6.23, its proof is similar to the one in loc. cit. and is left to the reader.

Theorem 6.25 Let $T$ be a locally compact topological space. If $f: U \times T \rightarrow \mathbb{R}$ is a function such that $f(\cdot, t)$ is weakly concave for each $t \in T$ and $f(x, \cdot)$ is continuous for each $x \in U$, then $f$ is continuous on $U \times T$.

Acknowledgements We are grateful to the anonymous referee for all her/his constructive remarks.

Funding Open Access funding provided thanks to the CRUE-CSIC agreement with Springer Nature.

Open Access This article is licensed under a Creative Commons Attribution 4.0 International License, which permits use, sharing, adaptation, distribution and reproduction in any medium or format, as long as you give appropriate credit to the original author(s) and the source, provide a link to the Creative Commons licence, and indicate if changes were made. The images or other third party material in this article are included in the article's Creative Commons licence, unless indicated otherwise in a credit line to the material. If material is not included in the article's Creative Commons licence and your intended use is not permitted by statutory regulation or exceeds the permitted use, you will need to obtain permission directly from the copyright holder. To view a copy of this licence, visit http://creativecommons.org/licenses/by/4.0/.

\section{References}

1. Allerman, L., Rau, J.: First steps in tropical intersection theory. Math. Z. 264, 633-670 (2010)

2. Botero, A., Gil, J.I.: Burgos: Toroidal b-divisors and Monge-Ampère measures. Math. Z. 300, 579-637 (2022) 
3. Boucksom, S., Favre, C., Jonsson, M.: A refinement of Izumi's theorem, Valuation theory in interaction, EMS Ser. Congr. Rep. Eur. Math. Soc. 55-81 (2014)

4. Jonsson, M.: Singular semipositive metrics in non-Archimedean geometry. J. Algebraic Geom. 25, 77-139 (2016)

5. Bourbaki, N.: Éléments de mathématique. Topologie générale. Chapitres 1 à 4. Hermann (1971)

6. De Loera, J. A., Rambau, J., Santos, F.: Triangulations. Structures for algorithms and applications, Algorithms Comput. Math. vol. 25, Springer-Verlag (2010)

7. Fulton, W.: Introduction to toric varieties, Ann. Math. Stud., vol. 131, Princeton Univ. Press (1993)

8. Fulton, W., Sturmfels, B.: Intersection theory on toric varieties. Topology 36, 335-353 (1997)

9. Gross, A.: Intersection theory on tropicalizations of toroidal embeddings. Proc. Lond. Math. Soc. 116, $1365-1405$ (2018)

10. Luukkainen, J., Väisälä, J.: Elements of Lipschitz topology. Ann. Acad. Sci. Fenn. Ser. A I Math. 3, 85-122 (1977)

11. Rau, J.: Intersections on tropical moduli spaces. Rocky Mountain J. Math. 46, 581-662 (2016)

12. Rockafellar, R.T.: Convex Analysis. Princeton Univ. Press, Princeton (1972)

Publisher's Note Springer Nature remains neutral with regard to jurisdictional claims in published maps and institutional affiliations.

\section{Authors and Affiliations}

\section{Ana María Botero ${ }^{1}$ • José Ignacio Burgos $\mathrm{Gil}^{2}$ • Martín Sombra ${ }^{3,4,5}$}

$凶$ Martín Sombra

sombra@ub.edu

http://www.maia.ub.edu/ sombra

Ana María Botero

Ana.Botero@mathematik.uni-regensburg.de

https://homepages.uni-regensburg.de/ boa15169/

José Ignacio Burgos Gil

burgos@icmat.es

http://www.icmat.es/miembros/burgos

1 Fachbereich Mathematik, Universität Regensburg, Universitätsstr. 31, 93053 Regensburg, Deutschland

2 Instituto de Ciencias Matemáticas (CSIC-UAM-UCM-UCM3), Calle Nicolás Cabrera 15, Campus UAB, Cantoblanco 28049, Madrid, Spain

3 Institució Catalana de Recerca i Estudis Avançats (ICREA), Passeig Lluís Companys 23, 08010 Barcelona, Spain

4 Departament de Matemàtiques i Informàtica, Universitat de Barcelona, Gran Via 585, 08007 Barcelona, Spain

5 Centre de Recerca Matemàtica, Edifici C, Campus Bellaterra, 08193 Bellaterra, Spain 